\title{
Textkritische Studien zum Buche Job.
}

Von Georg Beer in Halle a. S.

(Schluss.)

\section{Kap. XXXI.}

I. $*$ testamentum feci oculis meis $\mathfrak{h}^{2} *$ et non cogitabo

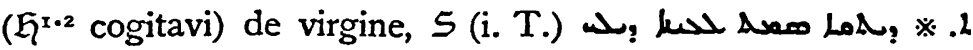

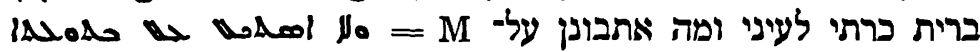

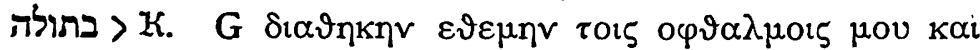

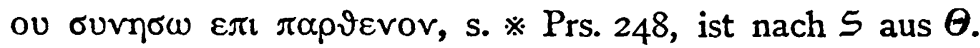

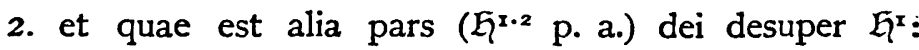
$\mathcal{L}^{2} *$ et hereditas omnipotentis de excelsis, 5 (i. T.) $10020 \%$ ומה חלק הלוה ממעל

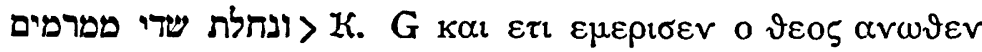

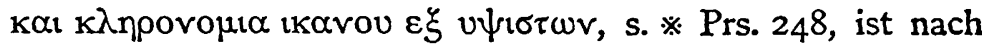
$\boldsymbol{S}$ aus $\boldsymbol{\theta}$. $\mathfrak{h}$ et quae $=\mathrm{S}^{\text {c.a }} \mathrm{k \alpha \imath} \tau \boldsymbol{\tau}$. $\mathfrak{\mathcal { G }}$ alia pars ist nach $\mathrm{M}$ חై übersetzt.

3. $\mathfrak{L}^{2} \approx$ (,wo $\approx$ fraglich" Lag.) nonne perditio erit iniquo

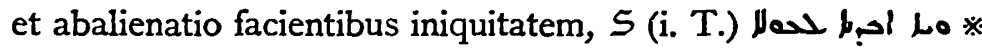

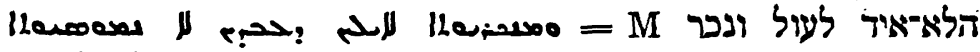

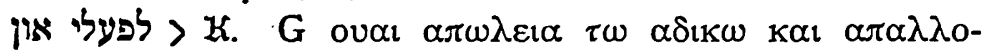

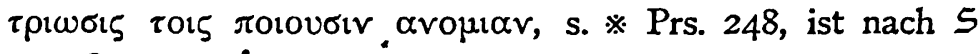
aus $\Theta$.

I7. ${ }^{2}$ ex eo: $S$ (i. T.) $)^{x}$ ow ממנה > G K.: C Sc.a 23 Prs. Compl. Ald. $\varepsilon \xi$ aurov, s. $※$ Prs. 255, ist nach Cod. Ven. Marc. b (Klst.) aus $\boldsymbol{A}$.

18. quia $a b$ adolescentia mea $\mathfrak{G}^{x} *$ enutriebam quasi pater $\mathfrak{h}^{2} *$ et de ventre matris meae $\mathfrak{h}^{\mathrm{x}} *$ dux eis fui: $\mathfrak{S}$

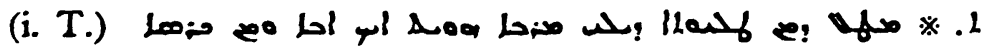
Zeitschrift E. d. alttest. Wiss. Jahrg. I8. II. 1898 . 
K > כי מנעורי גדלני כאב ומבטן אמי אנחנה

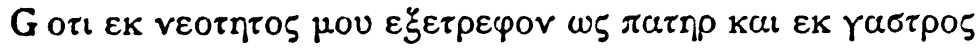

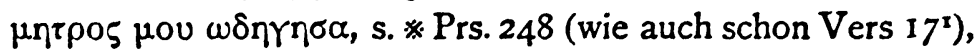
fehlt „Euseb. 1. cit. Cyrill. Alex. VI. part. i. p. 149“ Prs., ist nach $\kappa S$ aus $\Theta$. Ich nehme an, dass der $*$ in $\mathfrak{G}^{\mathbf{x}}$ vor enutriebam schon vor quia mitgehört.

19. $\mathfrak{h}^{2} *$ aut si despexi nudum pereuntem $\mathfrak{L}^{x} *$ et non operui $\mathfrak{G}^{2} *$ non habentem velamen $\mathfrak{h}^{x}: S$ (a. R.) \&l $\boldsymbol{l}^{2}$

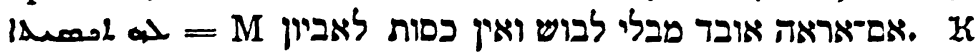

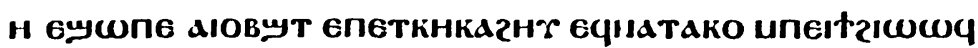

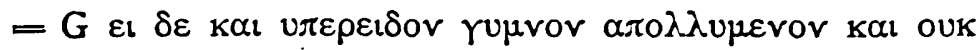

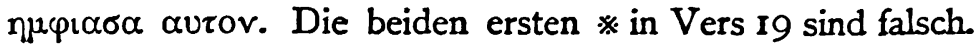

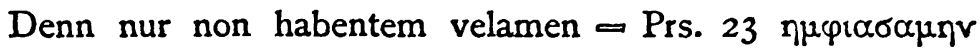

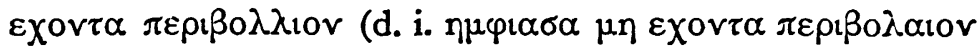
Fld.) > G K $S$ (i. T.) ist in $\mathfrak{G}$ ergänzt vgl. $S$. In $\mathfrak{L}$ fehlt aurov wie in A 22 Prs. Compl. Ald.

$21 .{ }^{\mathrm{x}} *$ super pupillum $\left\langle\left(\checkmark>\mathfrak{G}^{2}\right), 5\right.$ (i. T.) $\rangle \stackrel{2 .}{2} *$ M על-יתום. K EXN oropфanoc. $K$ beweist nicht, dass

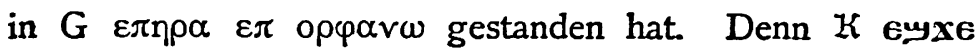

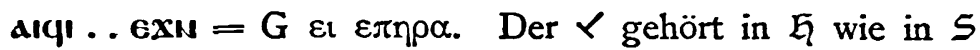

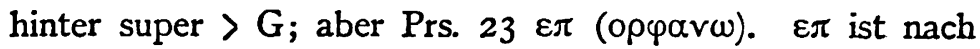
$S$ aus $A \Theta$.

23. ${ }^{2} \mathfrak{L}^{\mathrm{x}} \approx$ et a pondere eius non sustinebo, $S$ (i. T.)

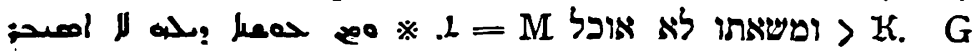

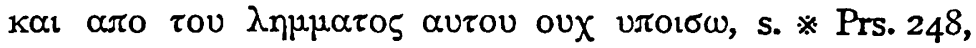
ist nach $S$ aus $\Theta$.

27. $\mathfrak{L}^{2} *$ et deceptum est clam cor meum $\mathfrak{q}^{x}: S$ (i. T.)

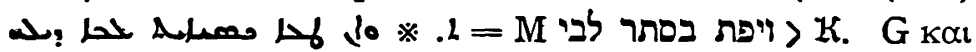

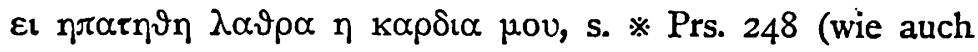
$27^{2}$ ), ist nach $S$ aus $\Theta$.

I Nach Cias. hat Prs. 248 erst $17^{2}$ sub $※$

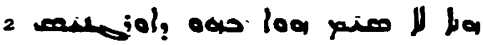


$35{ }^{1.2} \mathfrak{L}^{2} *$ quis mihi tribuat auditorem $\mathfrak{L}^{2} *$ manum

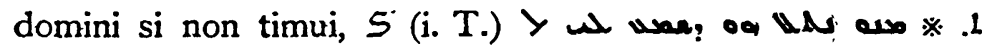

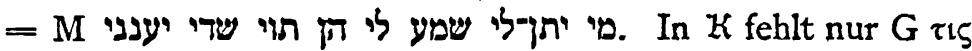

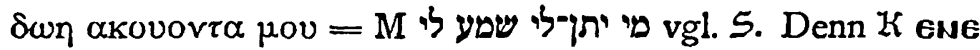

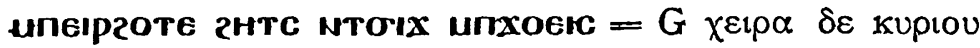

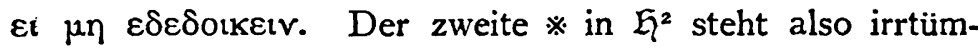
licherweise; beachte aber dass auch $\mathrm{k} 35^{2}$ wie $35^{\mathrm{x}} \mathrm{dem} \Theta$ zuspricht und nach Prs. bei Euseb. $35^{1.2}$ fehlt. Cias. giebt an, dass $35^{x}$ bei Prs. 248 s. $*$ stehe; nach Prs. hat Prs. 248 den $*$ vor Vers 34 .

Kap. XXXII.

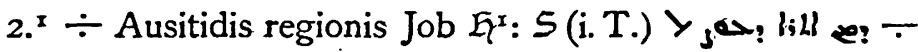

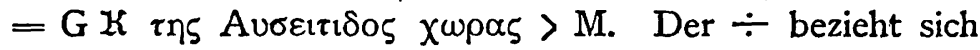
nicht mehr auf Job, denn dieses Wort kommt auch gleich in $M$, es ist in $\mathscr{E}$, gegen $G K S$, nur an falsche Stelle geschoben und durch Punkt von dem folgenden iratus autem est valde abgetrennt worden.

4. ${ }^{2} \mathfrak{L}^{\mathrm{x}} *$ quia (in $\mathfrak{K}^{\mathrm{x}}$, wahrscheinl. am Zeilenanf., steht eigtl. $*$ schon vor daret responsum) seniores se erant in

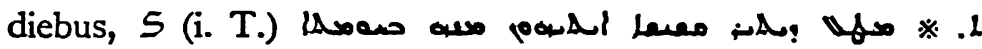

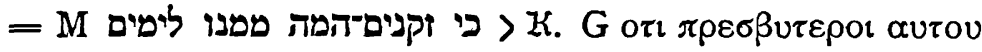

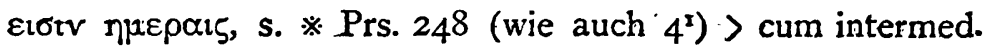

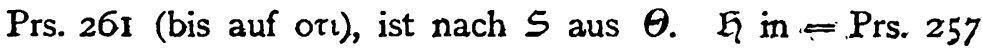
$\varepsilon v$ vor $\eta \mu$.

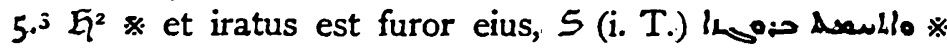

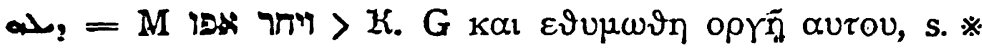
Prs. 248 (wie auch das Übrige von Vers 5), ist nach $\kappa S$ aus

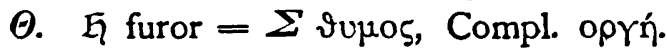

II. $\mathfrak{L}^{2} *$ ecce patiens fui in $\left(\mathfrak{G}^{3} \mathrm{ad}\right)$ verba vestra $\mathfrak{h}^{2} *$ et audivi vestram prudentiam usque quo discuteretis sermones,

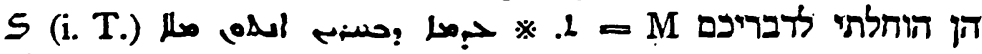
אמין עדתתבונתיכם עד־תחקרון טלין. In $k$ fehlt nur der in 5 s. * 
stehende Passus. G 5 hat als Vers $I 1 \varepsilon v \omega \tau i \zeta \varepsilon \sigma \vartheta \varepsilon \mu o v \tau \alpha$

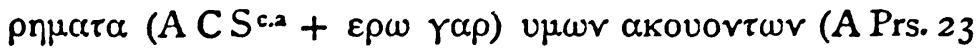

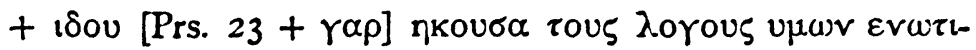

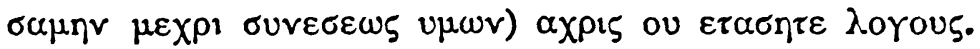

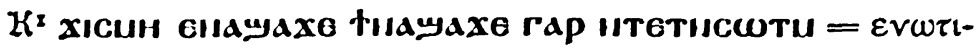

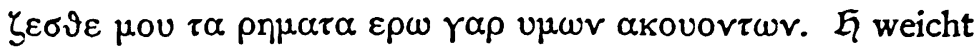
von $G K S$ ab. Sein Text ist aus $\Theta$ und $G(\Sigma)$ gemischt.

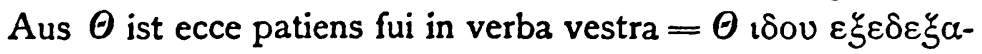

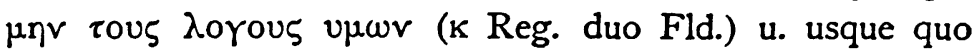

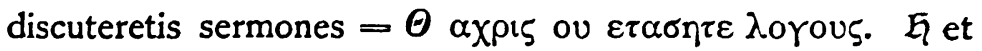

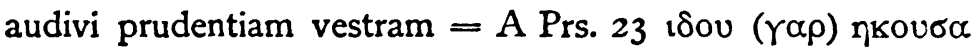

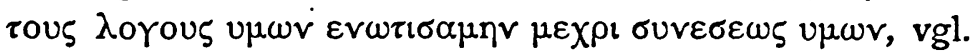

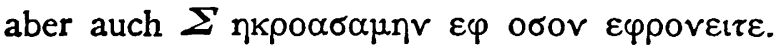

12. $\mathfrak{L}^{2} *$ et donec intellegerem vos: $\mathfrak{h}^{2} *$ et ecce non est qui arguat Job $\mathfrak{h}^{2} *$ et respondeat sermonibus eius ex vobis,

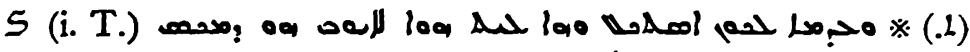
ויעדיכם אתבונן והנה אין לאיוב מוכיח

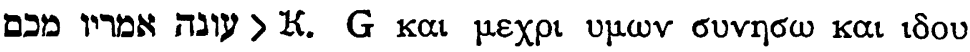

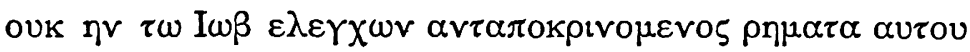
$\varepsilon \xi$ vpwr (Prs. 248 hat die Worte von $\alpha x \rho 15$ Vers II bis $\varepsilon \lambda \varepsilon \gamma(\omega)$ Vers 12 s. $*$ ) ist nach $\kappa S$ aus $\Theta$.

13. $\mathfrak{h}^{2} *$ ne dicatis invenimus sapientiam domini; $\mathfrak{h}^{2} *$ domi-

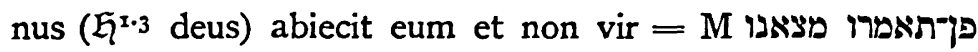

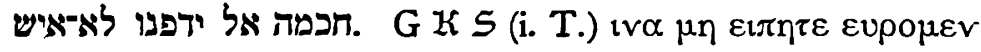

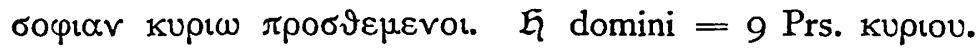
Was nach domini in $\mathfrak{F}$ steht, weicht von $G$ ab. $\mathfrak{L}$ schliesst sich hier an $\Sigma$ an, dessen Text in $S$ (a. R.) aufbewahrt ist

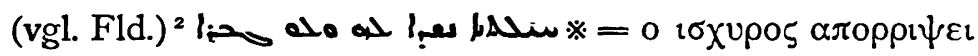

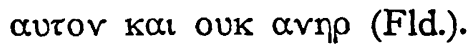

I hier beginnt erst Vers $I I$ in $K$. Bei Cias. sind von Vers 6 die Verse um eine $\mathrm{Zahl}$ yorgezählt.

2 darüber حله

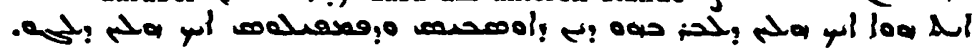


14. $\mathfrak{L}^{2} *$ necdum ad me locutus est $\mathfrak{L}^{2} *$ et in sermonibus vestris non respondebo ei = M ולא-ערך אלי מלין ובאמריכם לא של אשיבנו. GKS (i. T.) $\alpha \vee \vartheta \rho \omega \pi \omega \delta \varepsilon \varepsilon \pi \varepsilon \tau \rho \varepsilon \psi \alpha \tau \varepsilon \lambda \alpha \lambda \eta \sigma \alpha$

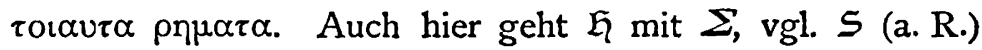
ist ov

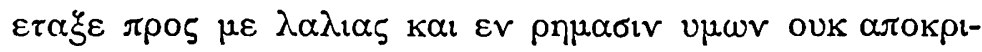

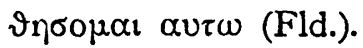

I 5. $\mathfrak{L}^{2} \approx$ obstupuerunt non responderunt ultra $\mathfrak{L}^{2} *$ defecerunt ab eis sermones = M לאו לאעו עוד העתיקו מהם מלים

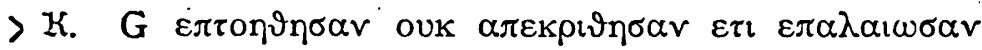

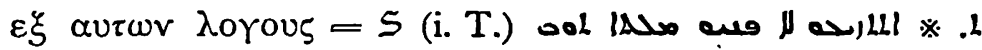
احلمه مسهر, ist nach $\kappa S$ aus $\Theta . \quad \mathfrak{L}=\Sigma$ (vgl. 5 a. R.)

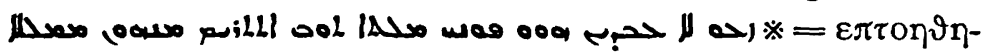

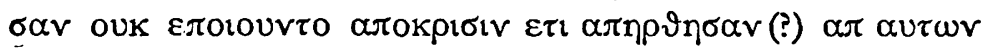

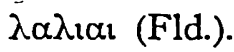

16. $\mathfrak{L}^{2} \approx$ quia ergo expectavi et non sunt locuti $\mathfrak{L}^{2} *$ et steterunt et non responderunt ultra $=M$ והוחלתי כי-לא ידברו

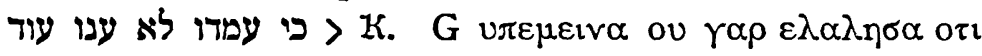

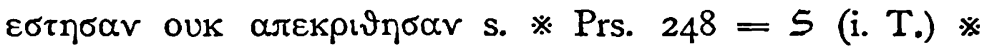
خ إist nach K 5

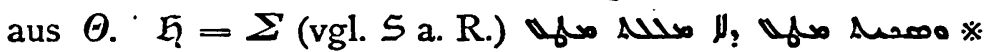
DoL إم

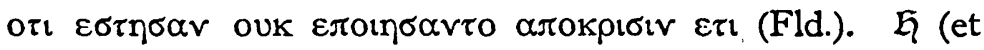
non) sunt locuti $=$ Compl. $\varepsilon \lambda \alpha \lambda \eta\rceil \sigma \alpha r$.

17. $\mathfrak{G}^{2} *$ respondebo $\left(\mathfrak{G}^{\mathrm{x} \cdot 3}\right.$ respondeam) et ego partem meam $\mathfrak{L}^{2} *$ et annuntiabo scientiam meam $\mathfrak{h}^{2} *$ homini vero permisistis loqui talia'verba $=M$ אענה אף־אני חלקי אהוה

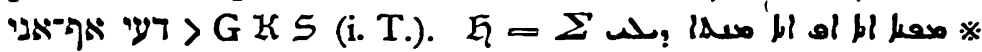
>

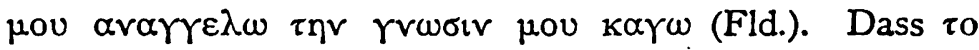

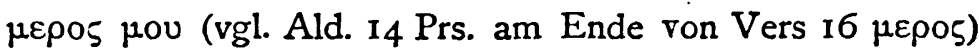
auf $\Sigma$ zurückgehe, besagen ausdrücklich $\kappa$ Cod. Ven. Marc. gr. XXI. saec. (Klst.). 


\section{Kap. XXXIII.}

$2 .^{2} *$ in gutture meo, $S$ (i. T.) $>$ (ب)

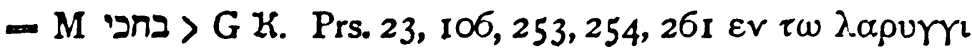
$\mu$ ov ist nach $S$ aus $\theta$.

8. * veruntamen dixisti in auribus meis, $S$ (i. T.) * .21.

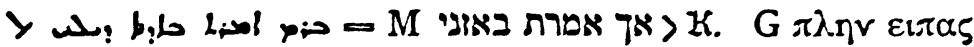
$\varepsilon \vee$ woเv $\mu$ ov ist nach $S$ aus $A \theta$, nach $\mathrm{k}$ nur aus $\theta$.

10. ${ }^{2} *$ sibi: $S$ (a. R.) $\div$ soul: $. \rightarrow=M$ is. $G$ hat nur

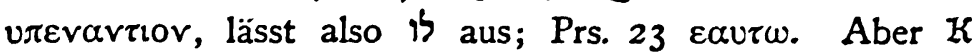
unetorbHB drückt is nach אויב aus.

$14 .^{2} *$ non considerabit ( $\mathfrak{G}^{\mathrm{x} \cdot 3}$ consideravit) illud $=\mathrm{M}$ לא ישורנה G K S (i. T.), kann nur aus $A$ oder $\Theta$ sein, da

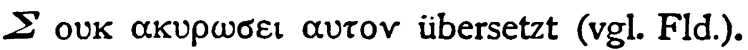

$20 .^{2} *$ et anima eius desiderabit cibum $\mathfrak{G}^{\mathrm{x}}: S$ (i. T.) $* .2$

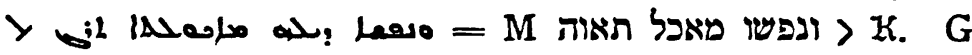

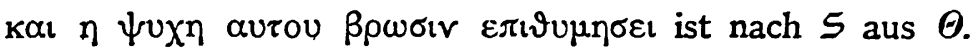

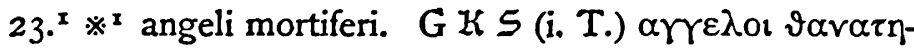
yopor übersetzt damit M מלאך מליץ. Der * hat keinen Sinn, vielleicht steht er für - und gehört vor mortiferi.

23. * non respondebit ei: > G K S (i. T.); aber Prs. 23

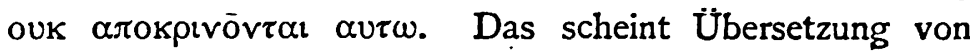
מליץ zu sein.

23 Ende. $\div$ et $\left(\right.$ et $>\mathfrak{L}^{\mathrm{x} \cdot 2}$ ) stultitiam suam $\mathfrak{L}^{\mathrm{x}} \dot{-}$ osten-

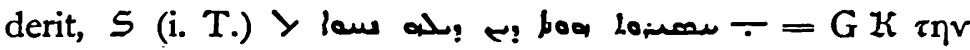

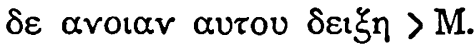

24. $\mathfrak{G}^{2} \div$ subveniet sibi ut non ( $\mathfrak{\complement}^{3}$ ne) cadat in morte et renovabit $\mathfrak{L}^{2} \div$ corpus suum sicut lituram ( $\mathfrak{\{}^{\mathrm{I} \cdot 2}$ litura)

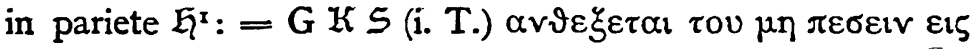

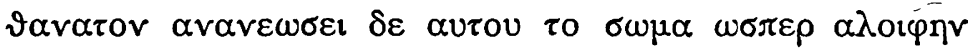
$\varepsilon \pi$ rotxou $>M$, oder ist nur eine freie erweiternde Wiedergabe Ms.

I $\mathfrak{G}^{\mathrm{x}}$ „nicht $※$, sondern $\times$ mit einem Striche darüber“ Lag. 
28. $*$ salva animam meam ne veniam in corruptionem $\mathfrak{h}^{2}$

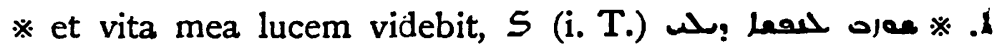

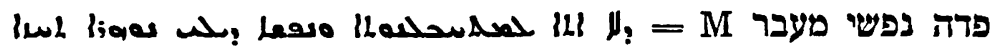

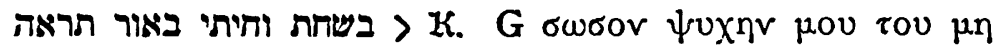

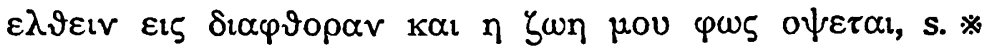
Prs. 248, ist nach $\kappa S$ aus $\Theta$.

29. $\mathfrak{L}^{2} *$ ecce haec omnia faciet deus $\mathfrak{h}^{2} *$ vices tres

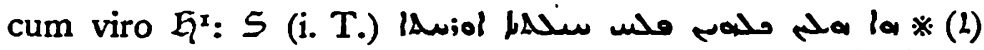

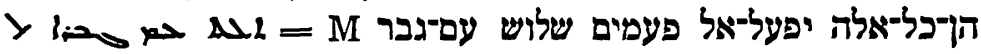

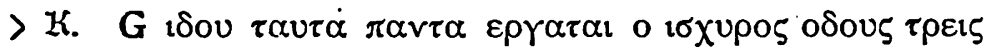

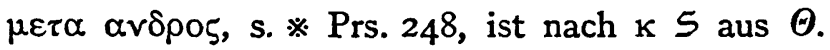

3 I. $^{2} \mathfrak{L}^{\mathrm{x}} *$ tace et ego loquar, $S$ (i. T.) - Al bo w

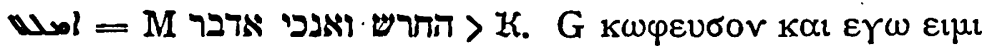

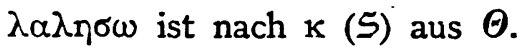

32. $\mathfrak{L}^{2} *$ si est tibi sermo responde mihi: $\mathfrak{L}^{2} *$ loquere

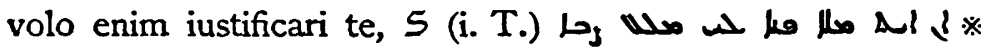

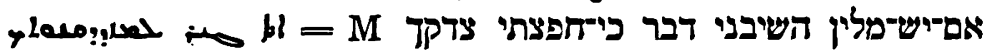

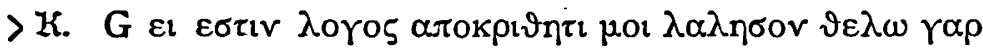

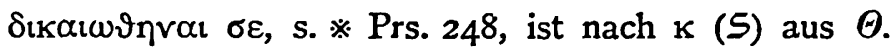

33. $\mathfrak{L}^{2} *$ alioquin tu (tu $>\mathscr{L}^{\mathbf{1} \cdot 2}$ ) audi me tace et docebo

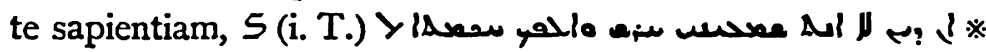

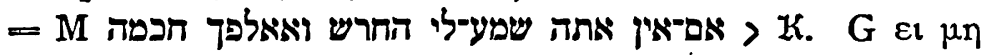

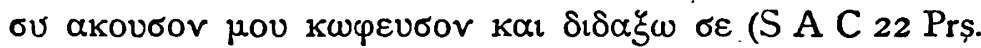
Compl. Ald. + бopı $\alpha$ ), s. $*$ Prs. 248 , ist nach $\kappa(5)$ aus $\Theta$.

\section{Kap. XXXIV.}

3. $*$ quia auris verba ( $\mathfrak{G}^{3}$ sermones) probat $\mathfrak{G}^{2} \approx$ et

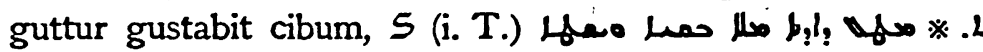

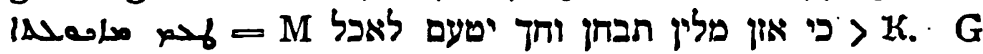

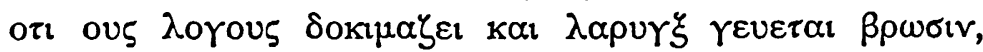
s. * Prs. 248 , ist nach $\times S$ aus $\theta$.

4. $\mathfrak{\zeta}^{2} *$ iudicium eligamus nobis sententiam inter nos-

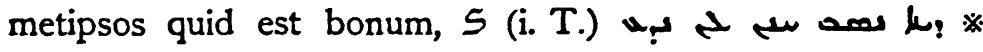




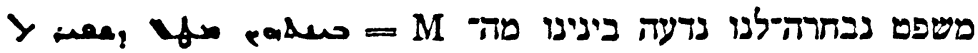

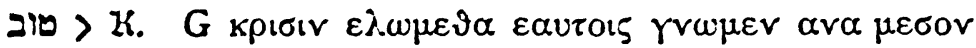

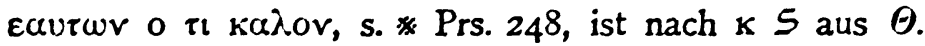

$6 .^{2} *$ violentum jaculum meum absque iniquitate, $S$ (i. T.) אגוש חצ בליפשע

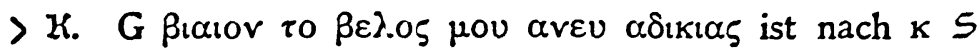
aus $\theta$.

7. $\mathfrak{L}^{2} *$ quis est vir ut Job $\mathfrak{G}^{2} *$ bibens subsannationem

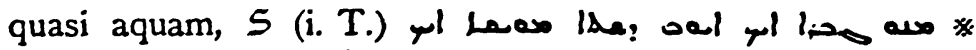

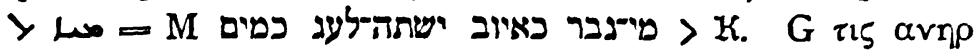

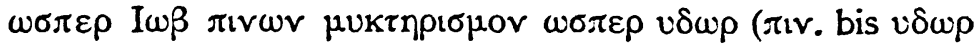
$>$ cum intermed. Prs. 26r) ist nach $\kappa S$ aus $\Theta$.

8. $\mathfrak{h}^{\mathrm{x}} *$ non peccans neque impie agens, $S$ (i. T.) $:-$

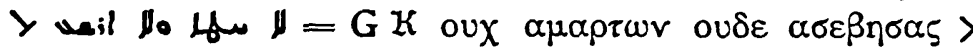

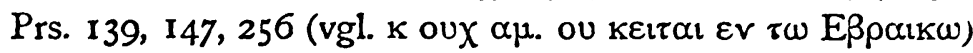
M. Der $*$ ist Fehler für -.

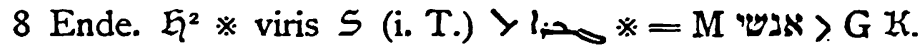

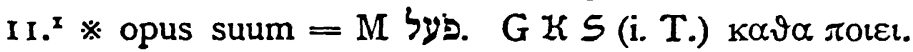
乌 übersetzt $M$ wörtlicher.

II. ${ }^{2}$ * et iuxta viam suam unus quisque repperiet $\mathcal{L}^{\mathrm{r}}$ :

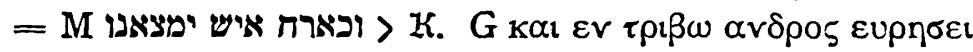
auror ist hexaplar. Text.

15. Ende $\div$ unde et (et $>\mathfrak{L}^{\mathrm{x} \cdot 2}$ ) formatus est, $S$ (i. T.)

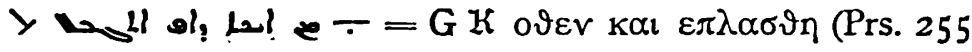
hat oงev $\mathrm{k \alpha l}$ s. $\div$ ) > M.

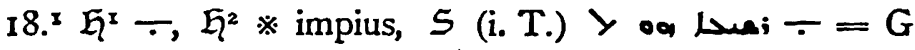
$\alpha \sigma \varepsilon \beta \eta \zeta>$ > K. Nur der - in $\mathfrak{L}^{\mathrm{I}}$ ist richtig.

18.2 * impiissime principibus =M M 1 K Chrys.

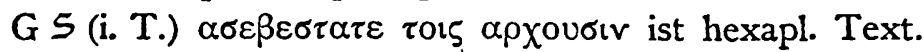

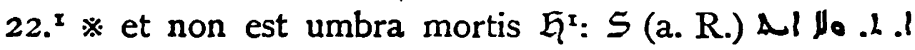

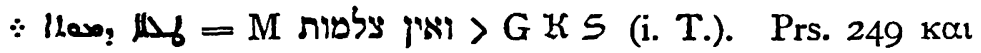

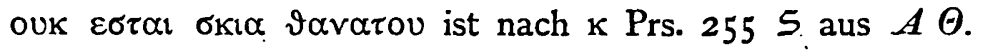
23: * quia non in homine positum est ultra: $S$ (a. R.) 
$\therefore 002$ 2

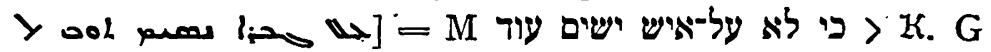

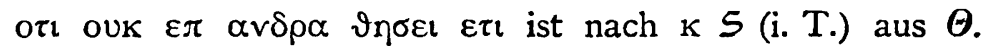
$\mathcal{L}$ giebt teilw. die Übersetzung von $\Sigma$ wieder ov $\gamma \propto \rho \varepsilon \pi$

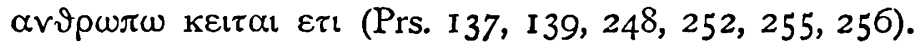

25. $*$ qui cognoscit opera eorum $\mathfrak{h}^{2} *$ et vertet $\left(\mathfrak{G}^{2}\right.$ evertet) noctem et humiliabuntur, $S$ (i. T.) W

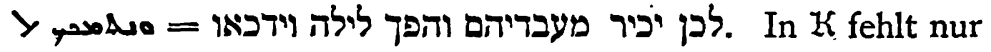
der in $\mathbf{S}$ s. $*$ stehende Passus, denn neteime enerzbнre

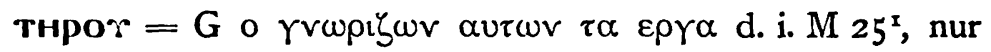
hat $\mathcal{K}$ тнроr als + gegenüber $G$. Der $※$ in $\mathfrak{L}$ vor $25^{x}$ steht also $\mathrm{zu}$ früh, freilich hat nach Montef. auch $\kappa$ hier schon ※. G $25^{2}$ ist nach $\kappa S$ aus $\Theta$. $S, 0$ d. $=$ Prs. 26I $\tau \alpha-$

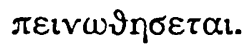

26. $\mathfrak{G}^{2} *$ et ex $\mathfrak{L}^{\mathrm{x}} *$ tinxit impios et gloriosos coram inimicis $\mathfrak{L}^{\mathrm{x}} *$ suis $\mathfrak{L}^{\mathrm{x}}:=\mathrm{M}$ תחתרשעים ספקם במקום ראים von

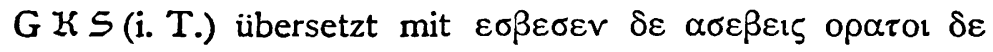

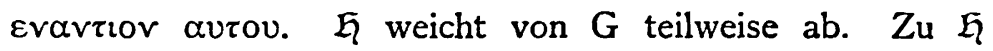

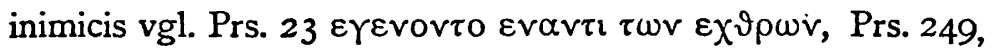

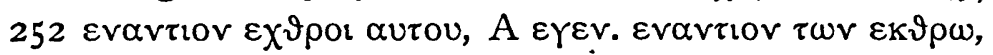

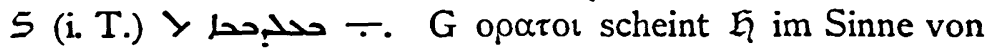
„,angesehen, sich rühmend“ aufgefasst zu haben. Der * steht nach Montef. auch in $\kappa$ vor Vers 26 , scheint aber wie in $\mathfrak{h}$ im Hinblick auf $S \mathbb{K}$ Irrtum zu sein.

27. quia declinaverunt a lege dei et iu $\mathfrak{L}^{2} *$ stitias eius

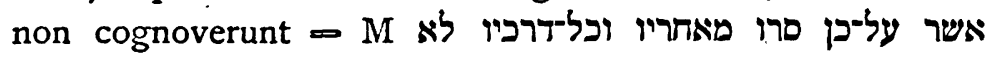

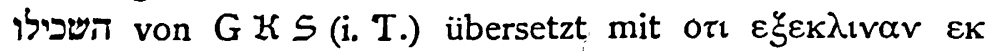

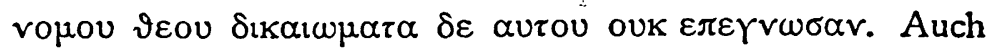
hier hat nach Montef. $\kappa$ vor Vers $27 *$, vgl. dazu Vers 26. 28. $\mathfrak{L}^{2} *$ ut perferatur ad eum $\mathfrak{L}^{x} *$ clamor pauperis et clamorem pauperum exau $\mathcal{L}^{\mathrm{x}} \% \operatorname{diet}, 5$ (i. T.)

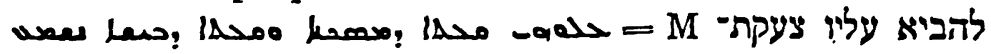

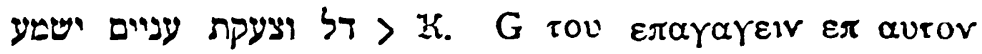




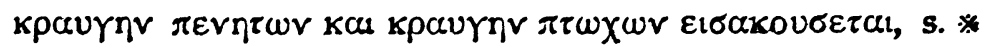
Prs. 248, ist nach $\kappa S$ aus $\Theta$. $\left\{\right.$ pauperis $=\operatorname{ACS}^{\text {ca }} 21$ Prs. Compl. Ald. $S$ revnros.

29. $\mathfrak{L}^{2} *$ ipse quietem dabit et quis poterit $\mathfrak{G}^{x} *$ condemnare ( $\mathfrak{q}^{x}$ commendare) abscondet faciem et quis vi $\mathfrak{h}^{x} *$ debit eum $\mathfrak{h}^{2} *$ et contra gentem et contra hominem $\mathfrak{h}^{x} *$

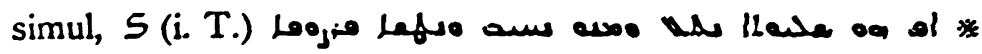

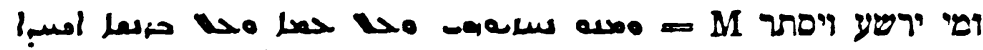

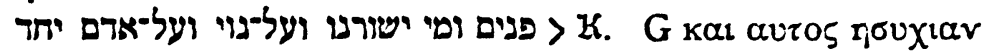

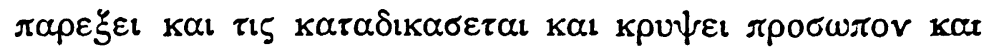

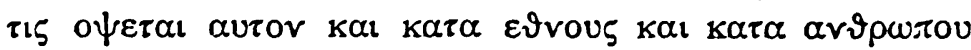
opov, s. * Prs. 248, ist nach $\kappa S$ aus $\theta$. In $\mathfrak{f}$ fehlt kat vor abscond. wie in Prs. 55.

30. $\mathfrak{L}^{2} *$ qui regnare facit hypo $\mathfrak{G}^{1} *$ critam propter per-

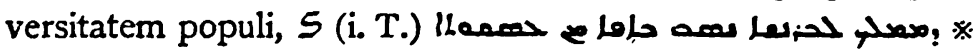

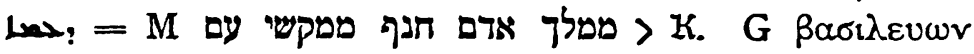

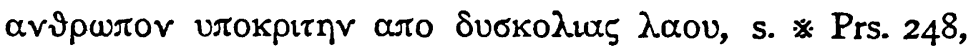
ist nach $\kappa S$ aus $\theta$.

31. $\mathfrak{h}^{2} *$ quia ad do $\mathfrak{h}^{\mathrm{x}} *$ minum loqui coepi non cessabo,

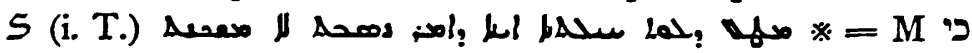

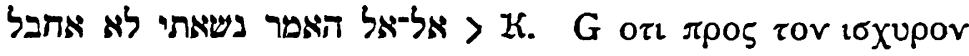
o $\lambda \varepsilon \gamma \omega r \varepsilon \mathfrak{l} \eta_{\eta} \varphi \alpha$ ouk $\varepsilon v \varepsilon \chi \cup \rho \alpha \sigma \omega$, s. ※ Prs. 248, ist nach $k$ $S$ aus $\theta$.

32. $\mathfrak{h}^{2} *$ at quod minus video, $\mathfrak{h}^{x}$ dafür absque me (me $\left.>\mathfrak{L}^{3}\right)$ vi $\mathfrak{h}^{x} *$ debo tu ostende mihi $\mathfrak{G}^{2} *$ si iniquitatem operatus

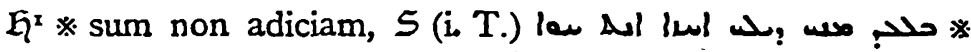
בלעדי אתוה אתה הרני אם עול M

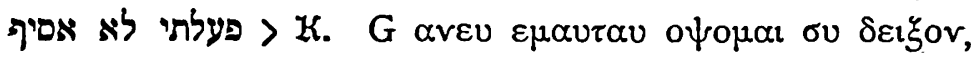

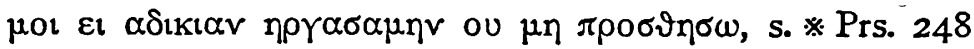
ist nach $\kappa S$ aus $\theta$.

33. $\mathfrak{L}^{2} *$ nunquid a te exiget eam $\mathfrak{L}^{\mathrm{x}} *$ quam reppulisti $\mathfrak{H}^{2} *$ tu enim elegisti et non ego: $*$ et si quid scis loquere

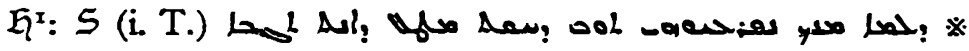




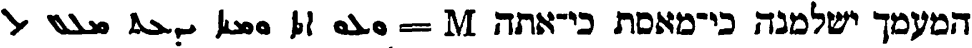

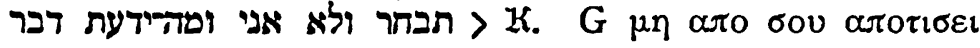

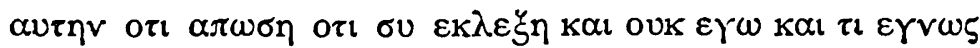
$\lambda \alpha \lambda$ пбоv, s. $*$ Prs. 248 , ist nach $\mathrm{K} S$ aus $\Theta$.

$34^{2} \mathfrak{h}^{2} \div$ et, $\mathfrak{L}^{\mathrm{x}} \div$ verbum meum $\mathfrak{L}^{\mathrm{x}}:=\mathrm{G} \mathbb{K} S$ (i. T.) $\delta \varepsilon ;=G K$ pov to $p \eta \mu \alpha, S$ (i. T.) $>$ w, $\mid \mathbb{A L}_{0}->\mathrm{M}$. Der $\div$ in $\mathfrak{h}^{2}$ steht $z u$ früh. $\delta \varepsilon>$ Chrys.

\section{Kap. XXXV.}

$2 .^{2} *\left(\mathfrak{h}^{x}\right.$ sogar hat $\left.2 *\right)$ tu quis es quia dixisti iustus sum $\mathfrak{F}^{\mathfrak{x}} *$ ante conspectum dei $=M$ אמרת צדקי מאל

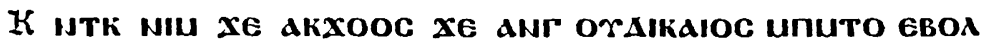
unxoeic, 5 -

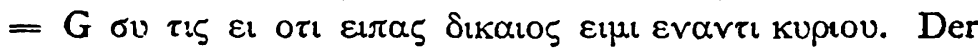
$*$ in $\mathfrak{G}$ ist Fehler für $\div$ u. sollte wie in 5 sich nur auf die Worte tu quis es (quia) $=\sigma u$ il $\varepsilon \imath$ beziehen, denen in $M$ nichts entspricht.

3. $\mathfrak{L}^{2} *$ aut dicis quid prodest tibi aut quid faciam si peccavi = M כי־תאמר מהייסכן־ד מה־אעיל מחטאתי. K H ekisaxooc de or ne nanobe nTalad $=\mathrm{S}^{\mathrm{c} . \mathrm{a}}$ A C 24 Prs.

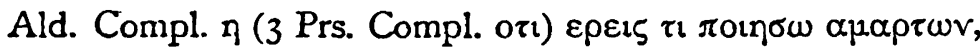
$S$ suld : of > G. In den griech. Codd., welche die Worte $\eta$ bis $\alpha \mu \alpha \rho \tau \omega r$ am Schluss von Vers 2

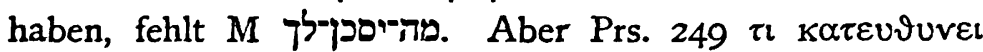

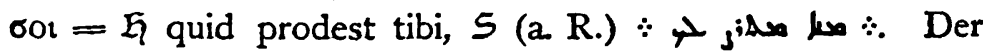
$*$ in $\mathfrak{G}$ sollte also sich nur auf die Worte quid prod. $t$. beziehen.

$7 .^{3} \mathfrak{L}^{2} *$ aut $\mathfrak{L}^{\mathrm{x}} *$ quid de manu tua accipiet, 5 (i. T.)

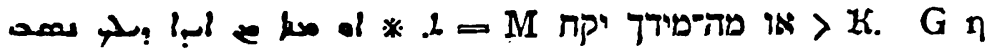

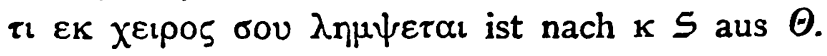

8. $\mathfrak{h}^{2} *$ viro simili. (战.2 similis) $\mathfrak{h}^{x} *$ tui impietas tua

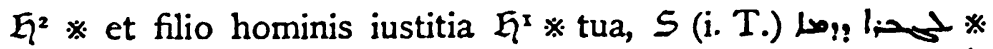

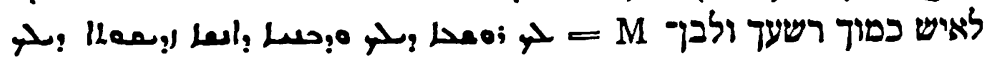




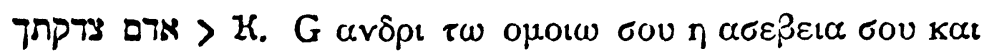

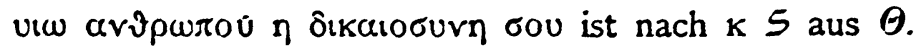

9. $\mathfrak{L}^{2} *$ a multitudine calumniantium cla $\mathfrak{L}^{1} *$ mabunt: vociferabuntur a brachio multorum, $S$ (i. T.) ILoL

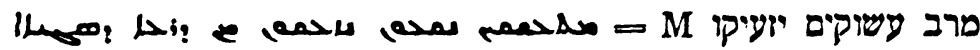

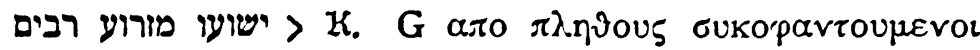

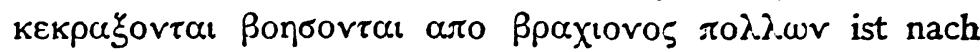

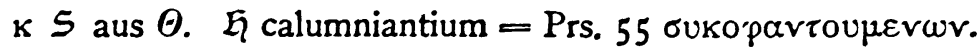

10. ${ }^{x}$ et non dixit ubi est deus qui fecit me, $S$ (i. T.) ולא־אמר איה אלוה

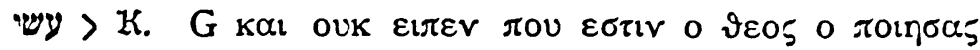
$\mu \varepsilon$ ist nach $\kappa S$ aus $\theta$.

I1. ${ }^{2}$ * sapientiorem me facit ( $\mathfrak{G}^{2}$ fecit), $S$ (a. R.)

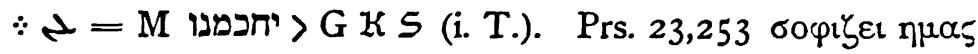
ist nach $\mathrm{k}$ Reg. un. aus $A \Theta$, nach 5 aus $A$. $\mathscr{q} \mathrm{me}$ !

12. $\mathfrak{L}^{2} *$ ibi clamabunt et non exaudies $\mathfrak{h}^{\mathrm{x}}$ : ( $\mathfrak{q}^{2}$ audiet, $\mathfrak{G}^{3}$ audies), 5 (i. T.) \ ueat لا

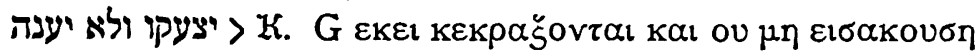
ist nach $S$ aus $\Theta$.

I 5. $\mathfrak{L}^{\mathrm{x}} *$ et nunc quia non est visi $\mathfrak{L}^{\mathrm{x}} * \operatorname{tans}$ iram suam $\mathfrak{L}^{2} *$ nec cognovit delicta vehe $\mathfrak{L}^{\mathrm{x}} *$ menter: $S$ (i. T.) $* . L$

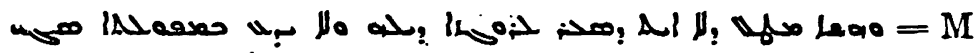

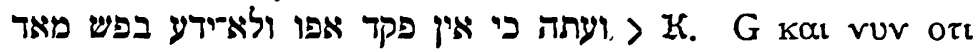

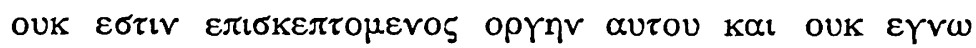

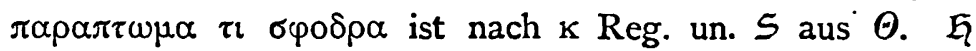
delicta $=\Sigma \pi \alpha \rho \alpha \pi \tau \omega \mu \alpha \tau \alpha$.

I6. et Job vane aperit os suum $\mathfrak{L}^{2} *$ in igno $\mathfrak{h}^{\mathrm{x}} *$ rantia verba multiplicat. $\mathfrak{h}^{x}: S$ (i. T.)

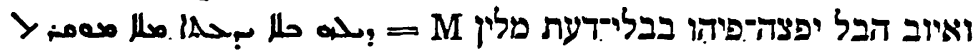

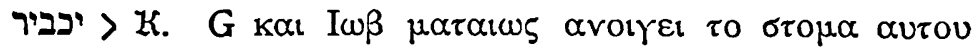

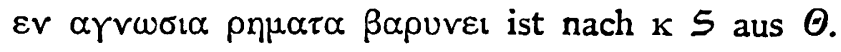


Kap. XXXVI.

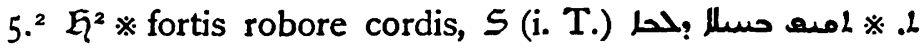

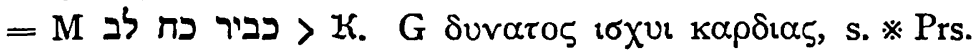
248, ist nach $\kappa S$ aus $\Theta$.

6. non vivificabit impium $*$ et iudicium pauperum dabit, 5 (i. T.) לאייחיה רשע

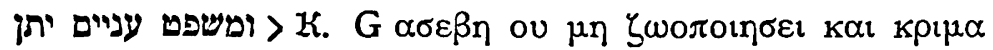
$\pi \tau \omega \chi \omega r \delta \omega \sigma \varepsilon 1$, s. * Prs. 248 , ist nach $\kappa 5$ aus $\Theta$.

7. non auferet a $\mathfrak{h}^{\mathrm{x}} *$ iusto oculos suos $\mathfrak{L}^{2} *$ et cum regibus in solio $\mathfrak{G}^{2} *$ et $\left(\right.$ e. $>\mathfrak{L}^{x}$ ) sedere $\mathfrak{L}^{\mathrm{I}} *$ eos facit in

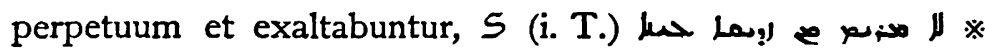

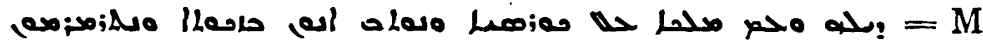
K.

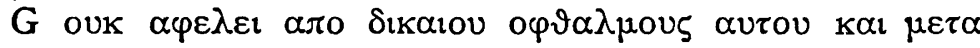

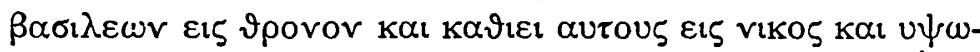

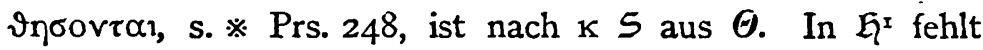
et vor sed. wie in S A 6 Prs. Compl. $\quad \mathfrak{L}$ in perpetuum = $\varepsilon 15 \tau \varepsilon \lambda 0513$ Codices Sergii (Prs.).

8. $\mathfrak{L}^{2} *$ et $\mathfrak{L}^{\mathrm{x}} *$ qui compediti sunt compedibus $\mathfrak{h}^{2} *$ capientur $\mathfrak{L}^{x} *$ in funibus paupertatis, 5 (i. T.) (20)

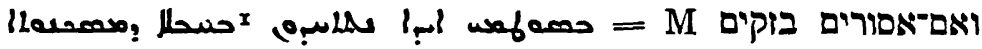

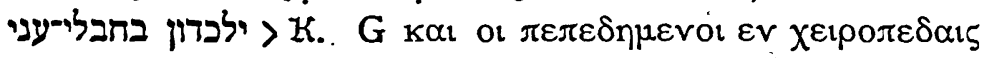

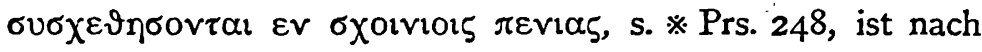
K 5 aus $\Theta$.

9. et annuntiabit eis $\mathfrak{G}^{\mathrm{x}}$ * opera eorum $\mathfrak{h}^{2} *$ et delicta cum fuerint roborati: 5 (i. T.) (1) ויגד להם פעלם ופשעיהם

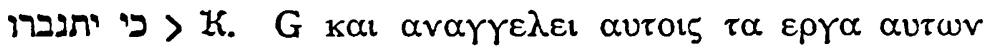

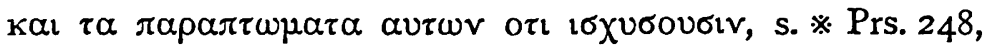

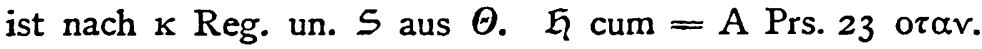
10. * sed iustum exaudiet $\mathscr{L}^{2} *$ et dixit quia ( $\mathfrak{L}^{\mathrm{x} \cdot 3}$ qui)

I a. R. cxolnioic. 
conver $\mathfrak{G}^{x}$ *tentur ab iniquitate: $=$ ויגל אונם למוסר ויאמר

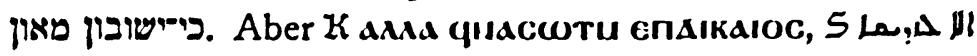

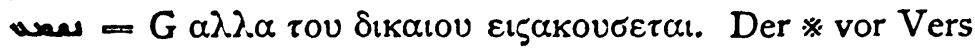
$10^{*}$ in $\mathfrak{L}$ ist also falsch. Erst $10^{2}>\mathcal{K}$ und steht bei $\mathcal{S}$ (i. T.)

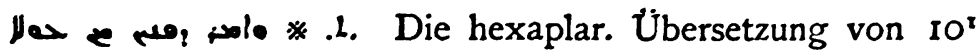

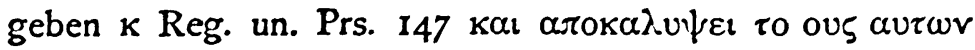

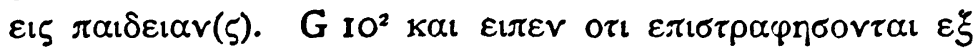
$\alpha \delta$ เkıร, s. * Prs. 248 (wie wohl auch 10), ist nach $\kappa$ Reg. un. aus $\theta$.

I1. $\mathfrak{L}^{2} *$ si audierint et servi $\mathfrak{h}^{\mathrm{x}} *$ erint $\mathfrak{L}^{2} *$ complebunt dies suos in bonis et annos suos in gloria, $S$ (i. T.) \* אعמح:

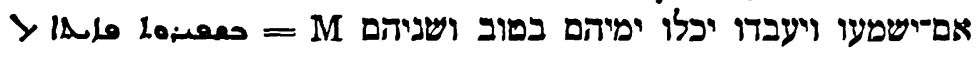

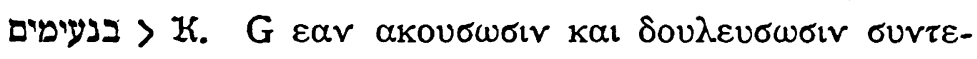

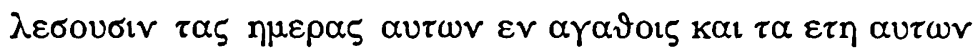

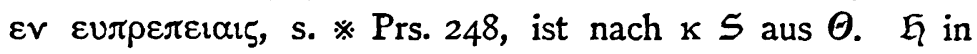

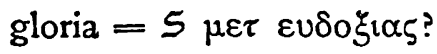

12. ${ }^{2}$ moneren $\mathfrak{h}^{\mathrm{x}} *$ tur inoboedientes erant. Der $*$, wohl am Zeilenanfang, geht auf Vers 13 .

13. $\mathfrak{L}^{2} *$ et hypocritae corde (co. $\left.>\mathfrak{h}^{\mathrm{x} \cdot 2}\right) \mathfrak{G}^{\mathrm{x}} *$ ponent furorem non clamabunt quia ligavit eos $\mathfrak{G}^{\mathrm{x}}: 5$ (i. T.) $* .1$

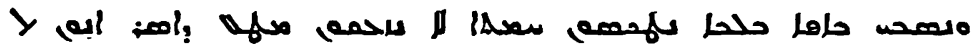

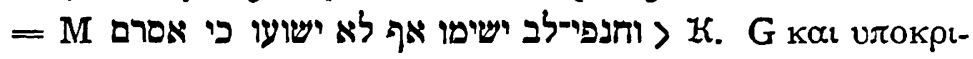

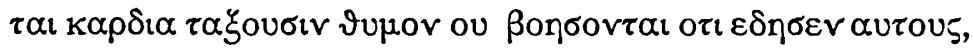
s. $*$ Prs. 248, ist nach (k Reg. un.) $S$ aus $\theta$.

16. $*$ et quia $\mathfrak{L}^{\mathrm{x}} \approx$ decepit te ab ore inimici abyssus $\mathfrak{G}^{2}$ * effusio ${ }^{2} \mathfrak{L}^{\mathrm{x}} *$ subter eam et $\mathfrak{h}^{2} *$ descendit mensa tua

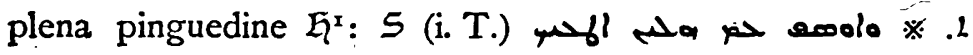

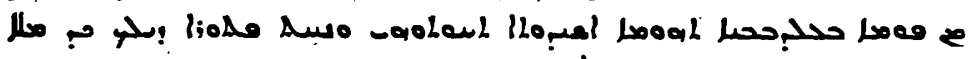

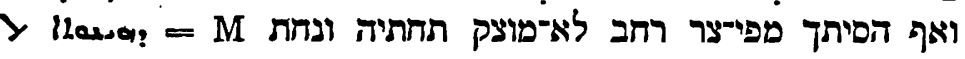

I $\mathfrak{G}^{\mathrm{r} \cdot 2}$ te et, $\mathfrak{E}^{3} \mathrm{ei}$, Augustin et (Casp.).

2 effusio, das Lag. vermutete, liest wirklich Eq3. Eq ${ }^{\cdot 2}$ Aug. effusi. 


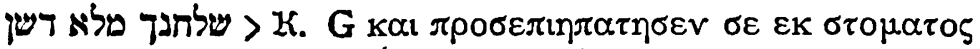

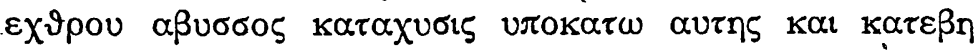

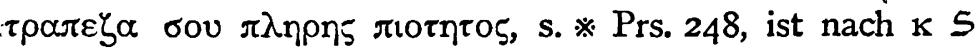
aus $\theta$. $\mathscr{L}$ et quia decepit $=$ Prs. 68, 137, 157, 161, 248,

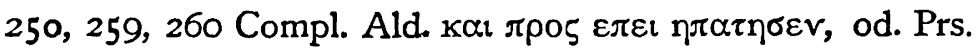

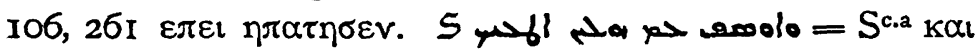
$\pi \rho о \sigma \varepsilon \tau \iota ~ \eta \pi \alpha \tau \eta \sigma \varepsilon v ?$

$20 .^{2} *$ ut ascendant populi pro $\mathfrak{L}^{\mathrm{x}} *$ eis: $\mathcal{S}$ (i. T.) $*$

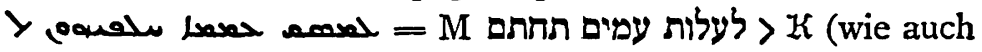

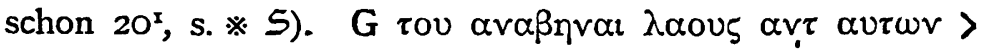
Prs. 249, ist nach $\kappa S$ aus $\Theta$.

21. $\mathfrak{L}^{2} *$ sed cura ne quid facias indecens $\mathfrak{h}^{2} *$ hoc enim השמר אל־תפן אל־און כי על־ה בחרת

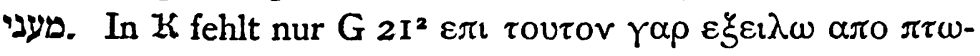

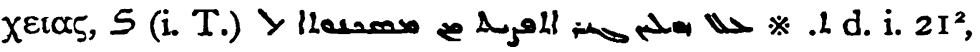
ist nach $S$ aus $\Theta$. Der $*$ vor $2 \mathrm{I}^{\mathrm{x}}$ in $\mathfrak{h}$ scheint also falsch zu sein. Zu beachten ist aber, dass G $2 I^{2} \alpha \lambda \lambda \alpha$ pvi $\alpha \xi \alpha \imath \mu \eta$ $\pi \rho \alpha \xi ั n S \alpha \delta$ in $\alpha$ in $\mathrm{A}$ u. ganz $2 \mathrm{I}$ in Prs. 249 fehlt. $\mathfrak{h}$ indecens $=\mathrm{S}^{\mathrm{c} . c} \alpha \tau$ \% $\alpha$.

$24 .^{2} *$ quem laudaverunt viri, $S$ (i. T.) of stel! var a $* .2$

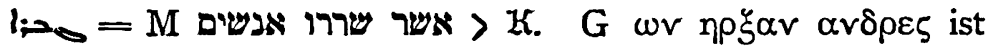
nach $\kappa S$ aus $\theta$.

25. $\mathfrak{L}^{2} *$ omnis homo respicit ad eum $\mathfrak{h}^{x}: 5$ (i. T.) $*$

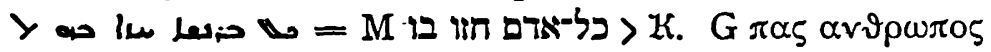
$\varepsilon \delta \varepsilon \vee \varepsilon \vee \varepsilon \alpha u \tau \omega$ ist nach $\mathrm{\kappa} S$ aus $\Theta$. $\mathfrak{h}$ ad eum $=S$ Prs. (55) $1.57,252$ Compl. 5 ev $\alpha u \tau \omega$.

26. $*$ ecce deus multus et nesciemus: $\mathfrak{L}^{2} *$ numerus annorum $h^{x} *$ eius infinitus, 5 (i. T.) (a הן־אל שגיז

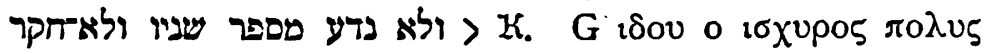

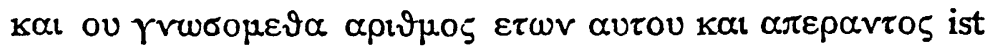
nach $\kappa S$ aus $\theta$. In $\mathcal{F}$ fehlt $\kappa \alpha$ vor $\alpha \pi \varepsilon \rho$. wie in Prs. 138,251 . 
27. $\mathfrak{L}^{2} *$ innumerabiles stillae pluviae $*$ effundetur imber per semitas suas =

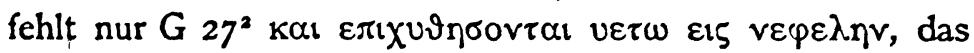

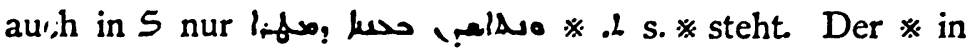

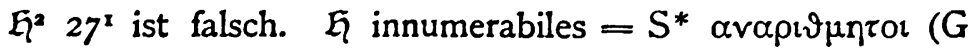

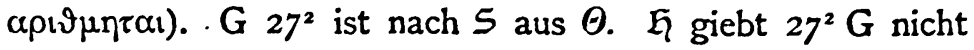
wörtlich wieder.

28. $\mathfrak{L}^{2} *$ fluent $\mathfrak{G}^{\mathrm{x}} *$ nubes et tenebrascent super homines pluri $\mathfrak{h}^{\mathrm{x}} *$ mos: $\div$ horam constituit iumentis et $\mathfrak{L}^{\mathrm{x}}-$ sciunt cubilis ordinem $\mathfrak{h}^{2} *$ in his omnibus non stu- $\mathfrak{h}^{\mathfrak{x}} \div$ pescit animus tuus $\mathfrak{h}^{x}: *$ nec mutatur cor tuum $\mathfrak{h}^{x} *$ a cor-

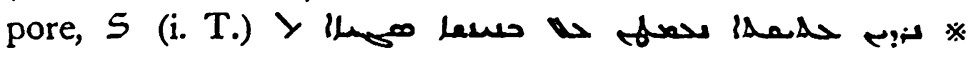
-

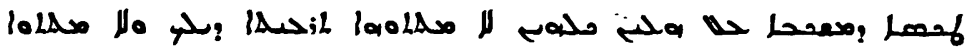

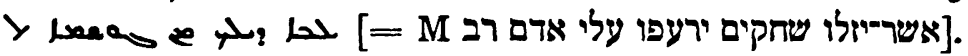
$\mathcal{K}$ übersetzt акгевс (lies атгевс Cias.) bis unєксcoud den

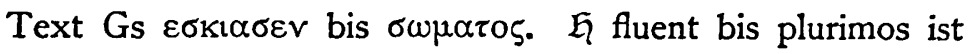

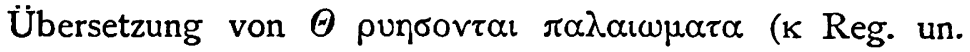

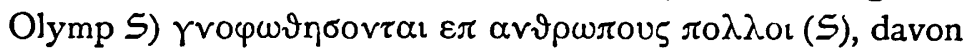
ist puns. $\pi \alpha \lambda$. in $G$ eingedrungen $>\mathcal{K}, \gamma \vee \circ \varphi \omega \vartheta$. bis $\pi \circ \lambda \lambda \circ$

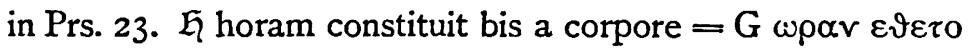

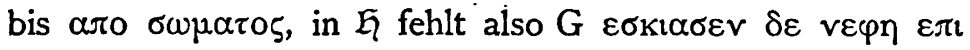

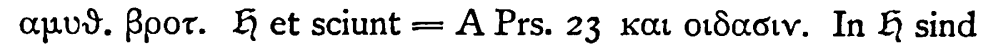
die $3 *$ von horam bis corpore falsch; der ganze Passus sollte wie in $\mathrm{k} \mathrm{S} \mathrm{s.} \mathrm{-} \mathrm{stehen.} \mathrm{G} \mathrm{\omega \rho ar} \mathrm{\varepsilon \vartheta \varepsilon \tau o} \mathrm{bis} \mathrm{Ende} 28>10$ Prs. Compl. (G $\omega \rho . \varepsilon \vartheta$. bis $3 \mathrm{I}^{\mathrm{I}}>$ Prs. 249), steht aber vor $37,5 .^{2}$

29. $\mathfrak{L}^{2} *$ si cogitaverit $\left(\mathfrak{L}^{\mathrm{I}} \mathrm{s}\right)$ extendere nebu $\mathfrak{L}^{\mathrm{x}} *$ lam $\mathfrak{L}^{2} *$ aeque ad tabernaculum expandit eam, 5 (i. T.) 10 $* .2$ אך הם אםיבין

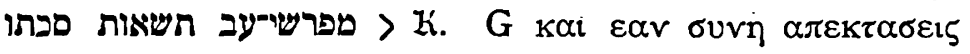

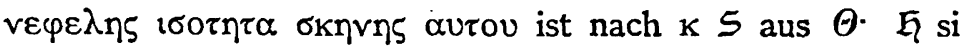

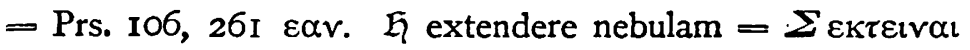
$\vee \varepsilon \varphi \varepsilon \lambda \eta \vee$ (Prs. 252); $\quad \mathfrak{Z} 29^{2}$ aeque bis eam giebt nur teil- 
weise $G$ wieder, schliesst sich weit mehr $\Sigma$ an, vgl. $\Sigma \varepsilon \xi$

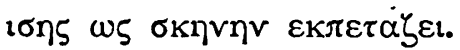

30. $*$ ecce effundit super eum lucem suam $\mathfrak{h}^{2} *$ et

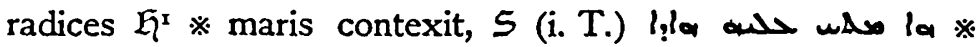

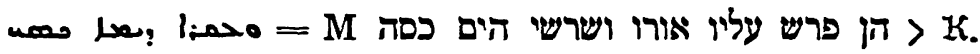

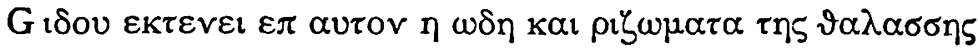
$\varepsilon \kappa \alpha \lambda \cup \psi \varepsilon v$ ist nach $\kappa S$ aus $\Theta$. $\{$ lucem suam $=\Sigma($ Fld.)

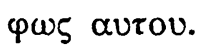

31. $\mathfrak{L}^{2} *$ in eis enim (en. $>\mathfrak{L}^{\mathrm{x} .2}$ ) iudicabit populos $*$ dabit

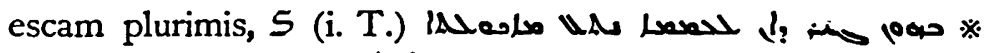

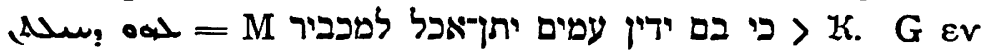

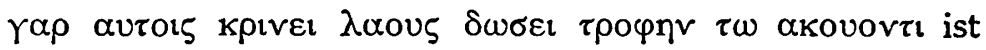

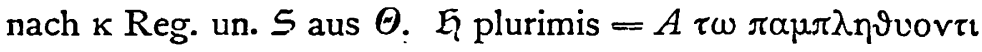

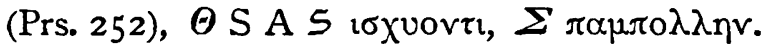

32. in manibus contexit $\mathfrak{L}^{x} \circledast$ lumen $\mathfrak{L}^{2} *$ et mandavit

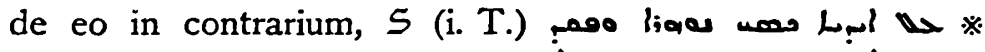

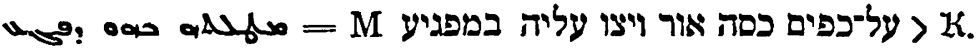

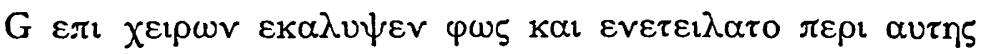
$\varepsilon \vee \alpha \pi \alpha \vee \tau \omega v \tau \imath$ ist nach $\kappa$ Olymp. Reg. un. 5 aus $\Theta$.

33. $\mathfrak{L}^{2} *$ ut $\mathfrak{L}^{\mathrm{x}} *$ nuntiaret super illo amico suo $\mathfrak{L}^{2} *$ possessio $\mathfrak{L}^{\mathrm{x}} *$ nem contra eum qui ascendere nititur $\left(\mathfrak{L}^{\mathrm{x}}\right.$

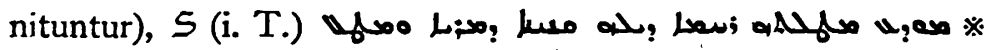

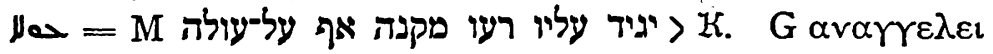

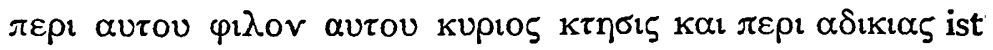
nach $\mathcal{S}$ aus. $\boldsymbol{\theta}$. In $\mathfrak{L}$ fehlt kupios wie in 18 Prs. Compl. $\mathfrak{L}$ contra eum qui ascendere nititur, vgl. $V$ et ad eam possit ascendere, schliesst sich an $M$ an, und leitet von y ab.

\section{Kap. XXXVII.}

I. $\mathfrak{L}^{2} *$ sed et $\mathfrak{h}^{1} *$ in hoc $\left(\mathfrak{G}^{2}\right.$ his) obstupuit cor meum $\mathfrak{F}^{2} *$ et evulsum est $\mathfrak{L}^{\mathrm{x}} *$ de loco suo, $\mathcal{S}$ (i. T.) l,a:0x $*$

1. 1,000

Zeitschrift f, d. alttest. Wiss. Jahrg. x8. II. 1898. 


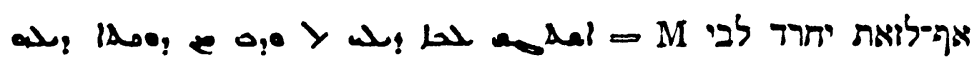

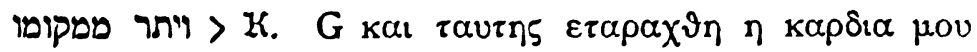

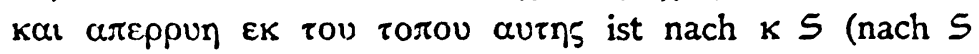
nur $1^{1}$ ) aus $\theta$. $\mathfrak{h}$ sed et $=A$ katre $\rho$ (Fld.)? $\mathfrak{L}$ in hoc - $A$ EV rourw.

2. $\mathfrak{L}^{2} *$ audite sonitum terroris et vocis eius (e. voc. ei.) $\mathfrak{G}^{\mathbf{x} \cdot 2} \mathfrak{L}^{2} *$ et $\mathfrak{L}^{1} *$ strepitum oris ipsius exeuntem, $\mathfrak{S}$ (i. T.)

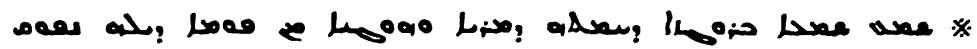

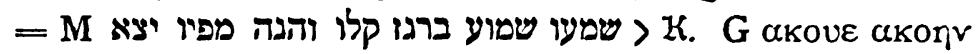

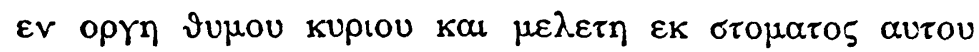
$\varepsilon \xi \varepsilon \lambda \varepsilon v \sigma \varepsilon \tau \alpha$ ist nach $\kappa S$ aus $\Theta$. $\mathfrak{L}$ audite ist nach $\mathrm{M}$

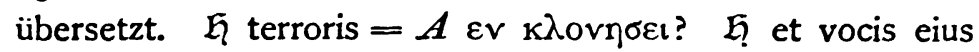

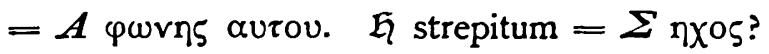

3. $*$ subter $\mathfrak{G}^{\mathfrak{x}} *$ universum caelum circuit $\mathfrak{h}^{2} *$ et lumen eius in finibus $\mathfrak{G}^{x} *$ terrae, 5 (i. T.) ILasi תחת כל-השמים ישרהו ואורו

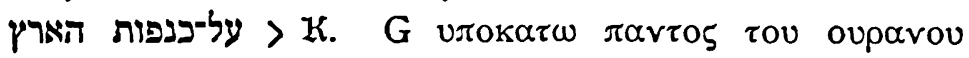

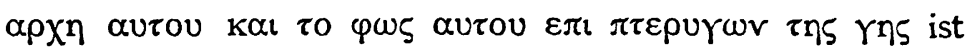

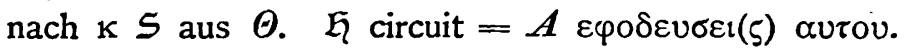

4. $\mathfrak{h}^{2} *$ post eum fremet vox tonabit in $\mathfrak{h}^{\mathbf{x}} *$ sonitu superbiae suae $\mathfrak{L}^{2} *$ et non poterit investigari cum audita fuerit

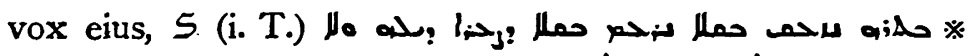

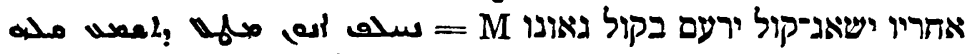

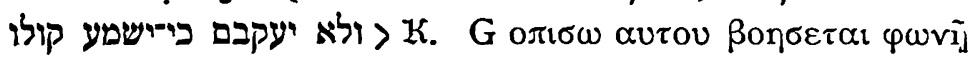

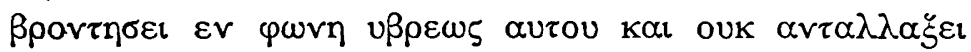

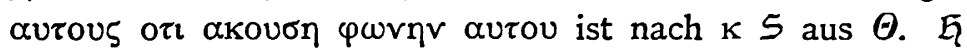

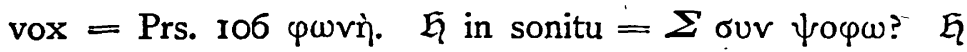
et non poterit investigari cum audita fuerit vox eis $=\Sigma \mathrm{Kal}$

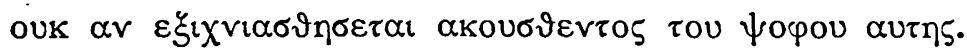

5. $\mathfrak{L}^{2} *$ tonabit fortis in voce sua mirabiliter $\mathfrak{L}^{\mathrm{x}}: \mathrm{S}$ (i. T.) ירעם אל בקולו נפלאות מ

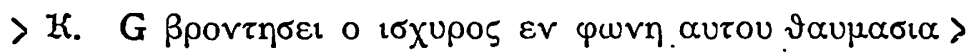
Prs. 55, ist nach $\kappa S$ aus $\Theta$. 
6. ${ }^{2} \mathfrak{L}^{\mathrm{x}} *$ et tempestas pluviae et tem $\mathfrak{G}^{\mathrm{x}} *$ pestas imbrium

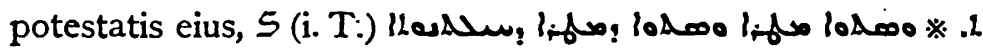

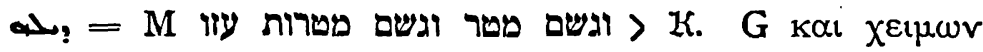

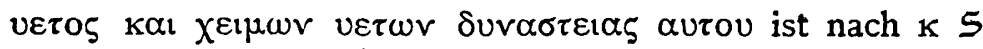
aus $\Theta$.

$7 .^{3}$ in manu omnis $\mathfrak{h}^{x} *$ hominis signat ${ }^{x}, S$ (i. T.) خاس.

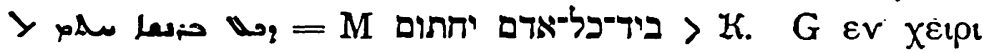

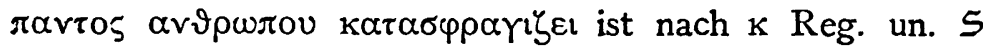
aus $\Theta$.

I0. $*$ et a spiritu dei dabitur glacies $\mathfrak{G}^{\mathrm{x}}:=\mathrm{M}$ מנשמת

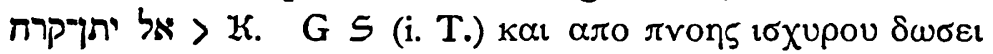
$\pi \propto$ Yoร, s. $*$ Prs. 248 (wie auch $\mathrm{IO}^{2}$ ), ist nach $\mathrm{\kappa}$ aus $\Theta$.

13. $\mathfrak{L}^{1} *$ sive in tri $\mathfrak{h}^{1} * \mathrm{bu} \mathfrak{G}^{2} *$ sive in terra sua $\mathfrak{h}^{2}$ * sive in misericordia inveni $\mathfrak{L}^{\mathrm{x}} *$ ri voluerit eam, $S$ (i. T.) אם -

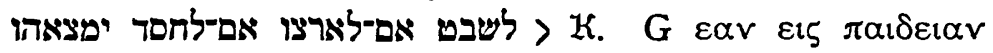

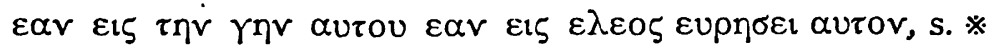
Prs. 248, ist nach $\mathcal{S}$ aus $\Theta$. $\mathfrak{L}$ in tribu $=\sum$ ei $\zeta \varphi \lambda_{\eta \eta}$ (Fld.)

I7. ${ }^{2} *$ ab $\mathcal{L}^{x}$ : austro, 5 (i. T.) > מדרום

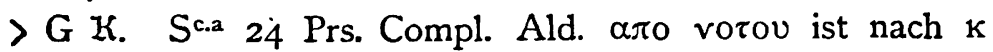
aus $\Theta$.

18. $*$ firmabis cum eo caelos qui aequaliter ad videndum fusi sunt, $S$ (i. T.) \רוקיע

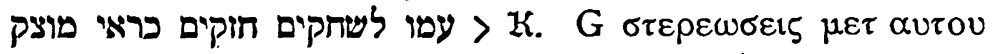

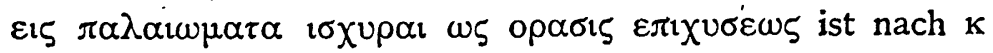

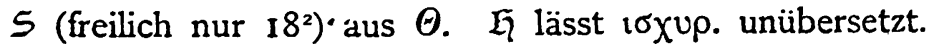

\section{Kap. XXXVIII.}

$2^{2}$. $\div$ et me putat latere, $S$ (i. T.) >

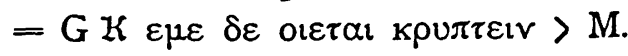

I $\mathfrak{Y}^{\mathrm{r} \cdot 2}$ signatur. 


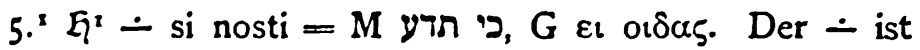
hier ein Irrtum.

7. ${ }^{2} \mathfrak{q}^{2} \div$ quando facta sunt simul. Es ist fraglich, auf welche Worte und wie weit der $\div$ sich beziehen soll. quando

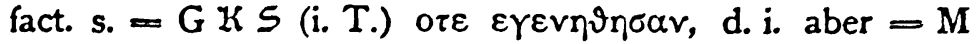
ברן (ברא). Vielleicht ist der $\div$ Versehen für * und sollte

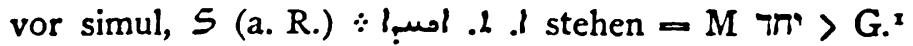

26. $\mathfrak{L}^{2} *$ ut et pluat super terram ubi non est vir $*$ in

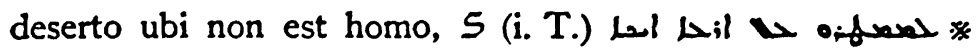

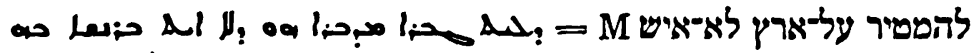

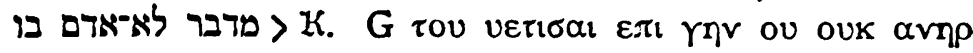

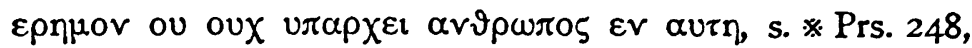
ist nach $\kappa S$ aus $\theta$.

27. $\mathfrak{q}^{2} *$ ut satiet invium et inhabitabile $\mathfrak{q}^{2} *$ et ut germinet

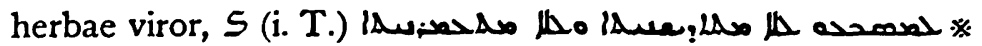

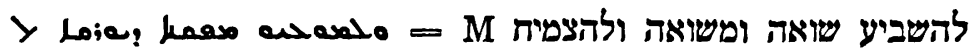

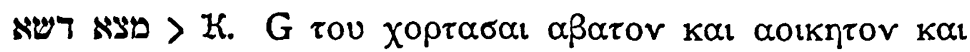

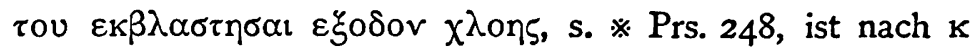
Reg. un. $S$ aus $\theta$.

32. $*$ an aperies Mazuroth in tempore suo et Vesperum super aedi $\mathfrak{Z}^{\mathrm{x}} *$ ficationem eius induces $\mathfrak{L}^{\mathrm{x}}: 5$ (i. T.) $* .2$

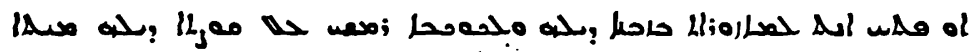

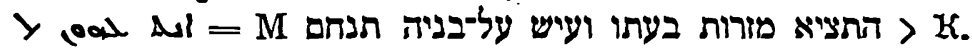

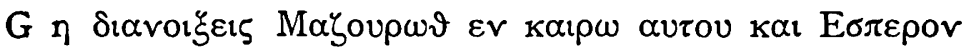

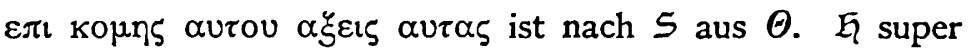

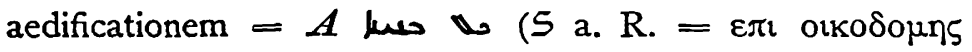
Fld.).

$37 .^{2}$ * et organa caeli in terram declinet. G $\mathcal{K} S$ (i. T.)

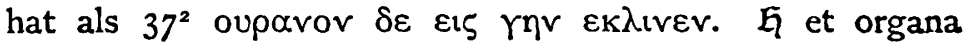

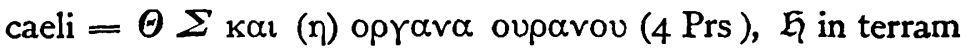

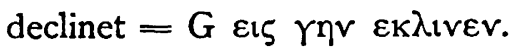

I $\{33$ bricht bei 38,16 mitten im Worte „fon“6 ab. 


\section{.. Kap. XXXIX.}

I. $\mathfrak{L}^{x} *$ si cognovisti tempus pariendi tragelaforum in

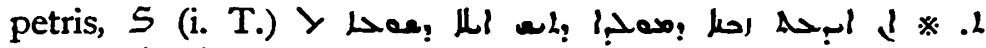

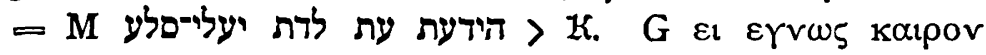

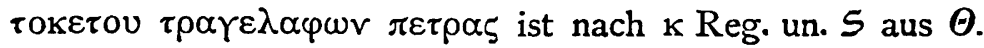
A hat $I^{2}$ als $3^{2}$.

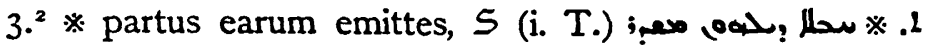

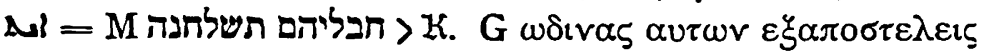
$>$ cum intermed. Prs. 26r, suppleta a diversa manu Prs. I47, ist nach Olymp. Prs. 137, 138, 139 5 aus $\Theta$. A hat $3^{2}$ als $3^{x}(\varepsilon \lambda v \sigma \alpha \zeta)$.

4. abrumpent $\mathfrak{G}^{x} *$ filii earum $\mathfrak{L}^{2} *$ multiplicabuntur in

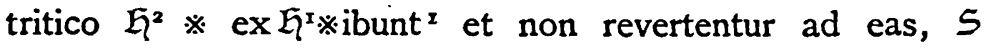

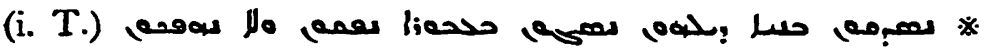

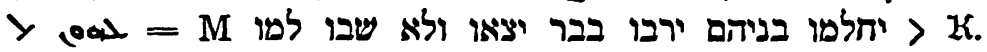

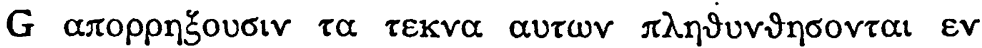

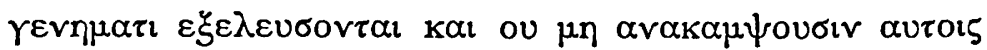

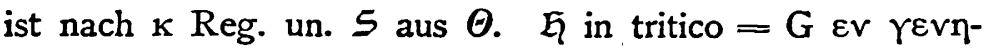

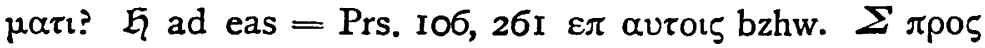
avras (Fld.).

6..$^{2}$ * et tabernacula $\left(\mathfrak{L}^{2}\right.$ habitacula) eius salsuginem $\mathfrak{h}^{x}$ :

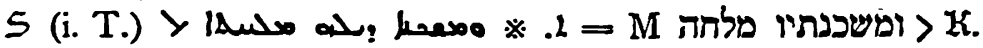

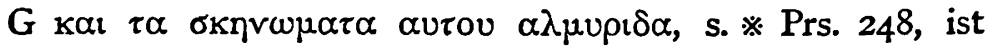
nach $\mathrm{K} S$ aus $\theta$.

8. $*$ consideravit montes pascuae suae $\mathfrak{h}^{2} *$ et post omne

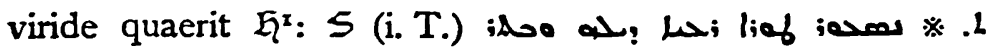

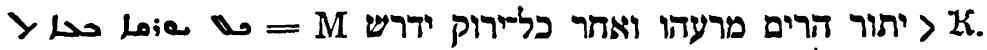

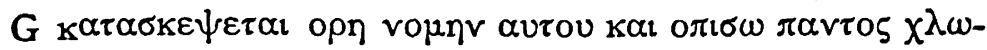
pov $\zeta \eta \tau \varepsilon^{i}$ ist nach $S$ aus $\theta$. $\quad \bar{q}$ scheint ropns gelesen $z u$ haben.

$x$ cf. Lag. 
123. * quia, S (a. R.) $\div$ al * oald. Fld. bezieht den * von $\mathfrak{h}$ darnach auf ei, vor quia, das hexapl. Ursprungs ist S" Prs. 23 aurw."

13. $\mathfrak{h}^{2} *$ penna $^{2}$ struthionum mix $\mathfrak{q}^{1} *$ ta est alis herodionis et accipitris, 5 (i. T.) هo

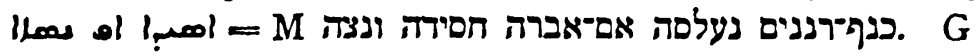

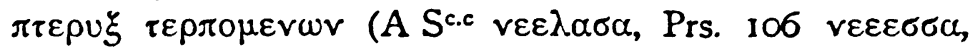

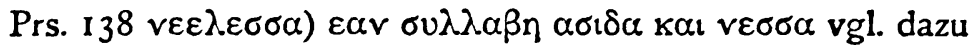
5 am ob. R. TEPY $\Xi$ TEPПOMENQN NEE $\Lambda \Sigma \Sigma A$ [sic] AN $\Sigma Y \Lambda \Lambda$ ABH A $\Sigma I \triangle A$ KAI NE $\Sigma \Sigma A$. $\mathfrak{h}$ struthionum =V struthionum, ist nach $M$ רנים ubersetzt. $\mathfrak{G}$ herodionis et accipitris

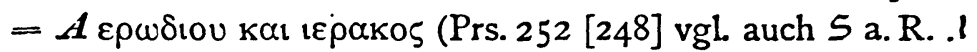

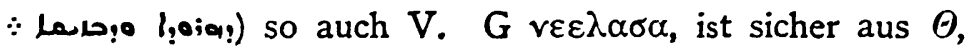
zu dessen Übersetzungsart die Beibehaltung unverstandner hebr. Wörter gehört. G I $3^{2}$ ist nach 5 aus $\Theta$. $\{$ schliesst sich in $13^{2}$ in dem, was er nicht aus $A$ hat, nicht an $\Theta$, auch nicht an $\Sigma$ an, sondern ubersetzt nach eignen Kenntנעלסה אם-אברה zurückzugehen, indem $\mathfrak{h}$ Ms $\boldsymbol{~} \boldsymbol{N}=\boldsymbol{y}$ auffasste.

14. $\mathfrak{L}^{2} *$ et re $\mathfrak{L}^{1} *$ linquit in terra ova sua $\mathfrak{h}^{2} *$ et in

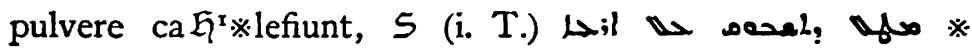

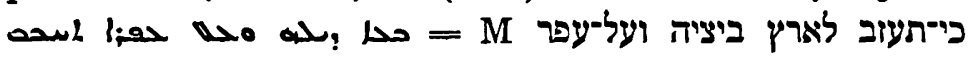

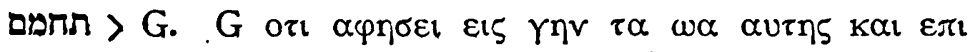
xouv $\vartheta \alpha \lambda \psi \varepsilon \varepsilon$ ist nach $S$ aus $\Theta$. $14^{2}-16>$ Chrysost. (Prs).

15. et oblita est quia pes dispergit ea $\mathfrak{L}^{\mathrm{x}} *$ et bestiae

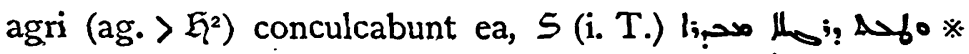

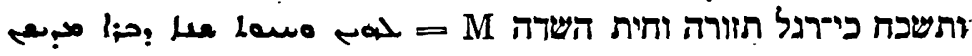

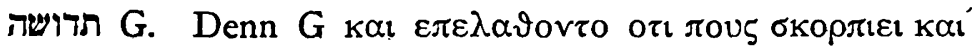

- I "In nostris codicibus sequitur lacuna inde a v. 9 huius capitis ad v. 8 a cap. XL" Cias. S. XXXII. Damit ist die Kontrolle für die $※$ in $\mathcal{K}$ bzhw. $S$ für Kap. $39,9-40,81$ verloren.

2 In $\mathcal{G}^{\mathrm{r}}$ steht der $※$ schon vor aream Vers 12 $^{2}$, wahrscheinlich am Zeilenanfange, bezieht sich also auf Vers 13. 


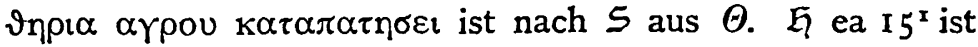
aus $A$ bzhw. $\Sigma$, vgl, auch 5 . $\{$ oblita est $=A \varepsilon \pi \varepsilon \lambda \alpha \vartheta \varepsilon \tau$ : $\mathfrak{h}$ ea $15^{2}=$ Prs. $23 \alpha u \tau \alpha$.

16. $\mathfrak{L}^{2} *$ obduru $\mathfrak{L}^{\mathrm{x}} *$ it contra filios suos ne sint eius $\mathfrak{L}^{2} *$ frustra labo $\mathfrak{L}^{\mathrm{x}} *$ ravit $\mathfrak{L}^{2} *$ absque ullo timore, $\mathcal{S}$ (i. T.)

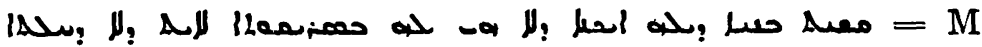

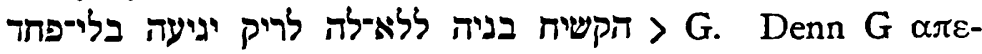

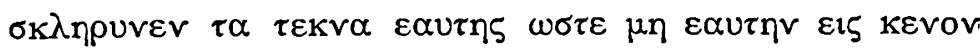

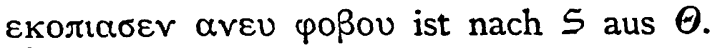

17. $\mathfrak{L}^{2} *$ quia despexit $\mathfrak{L}^{2} *$ eam dominus in sapientia $\mathfrak{L}^{2} *$ et non distribuit ei in $\mathfrak{h}^{2} *$ tellectum, 5 (i. T.) כי־השה

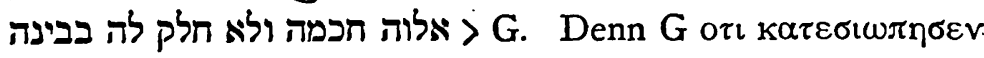

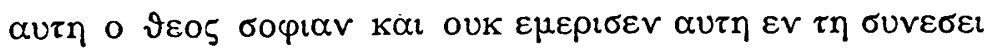
ist nach 5 aus $\Theta$. Bei Chrys. fehlt $17^{2}-18^{x}$.

18. $\mathfrak{L}^{2} *$ cum tempus fuerit in altum se levabit (eleva$\mathfrak{L}^{x} *$ bit) $\mathfrak{G}^{2} *$ et deridebit equitem et ascensorem $\mathfrak{h}^{x}: 5$

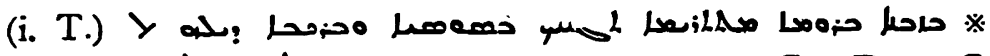

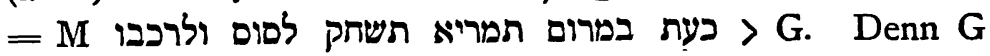

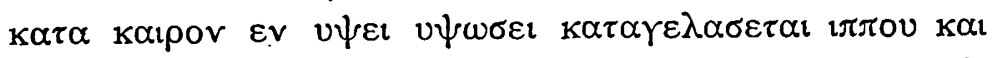

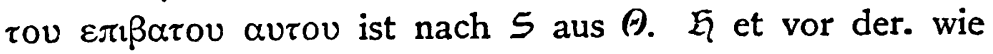
Prs. 161,248 Ald. Chrys. In $\mathfrak{h}$ fehlt aurou nach $\varepsilon \pi \_$. wie in Prs. 249.

$23^{2} *$ lanceae hastaeque cum tremore, 5 (a. R.) L L 2 * $* .2$

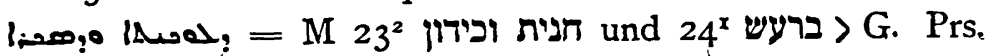

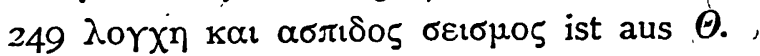

$26^{2} *$ et respiciens ad austrum, aber $S$ ohne Zeichen

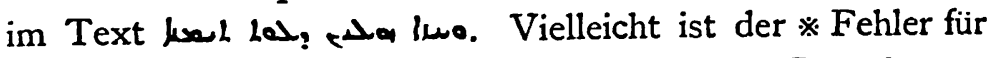

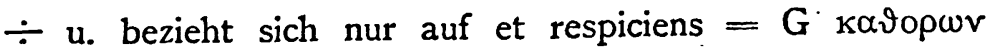
$>\mathrm{M}$.

$28 .^{2} *$ in summitate petrae et in caverna, 5 (i. T.) $* . L$

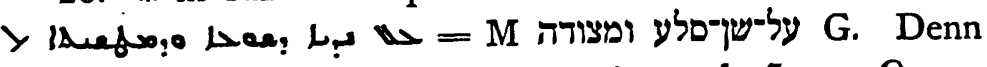

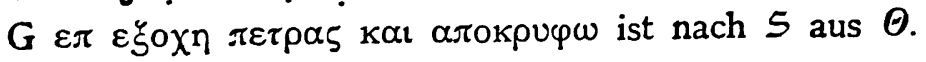


$29 .^{2} *$ longe oculi eius prospiciunt $\mathfrak{L}^{\mathrm{x}}: S$ (i. T.)

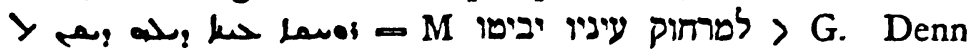

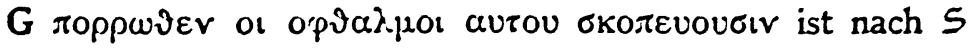
aus $\theta$.

\section{Kap. XL.}

$2 .^{2} \mathfrak{h}^{x} *$ arguens deum respondebit ita, 5 i. T. مص صan

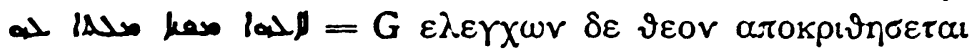
$\alpha \cup \tau \eta v$ hat $2^{2}$ nicht sub $*$ $\{\mathfrak{q}$ scheint hier gegenüber $S$ das Richtigere zu haben.

4. * quid ergo iudicor $\div$ commonitus et increpatus a domino $\mathfrak{L}^{2} \div$ audiens talia cum nihil $\operatorname{sim} \mathfrak{G}^{x}: \mathcal{S}$ (i. T.)

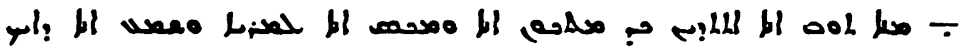

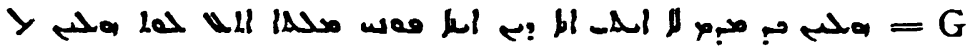

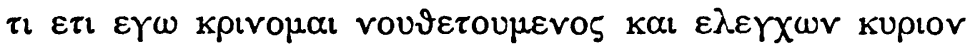

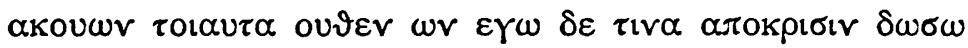
$\pi р о \varsigma \tau \alpha u \tau \alpha$. Dieser ganze Passus fehlt in M, bzhw. ist er eine freie Wiedergabe von Ms Text V. 2 u. 4. Vgl. $\tau$... . кptv.

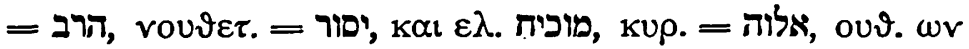

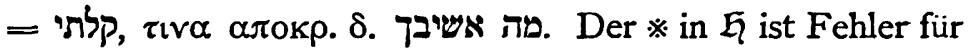
$\div$ Geht in Wirklichkeit der $\div$ in $\mathfrak{h}$ ursprünglich nur bis $\operatorname{sim}=\mathrm{G} \omega v$ ? Oder gehört auch manum ponam ad os meum noch hinzu?

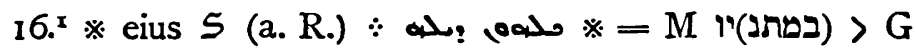
Aber sowohl $S$ (i. T.) als $K$ und Prs. 23, 249 aurov.

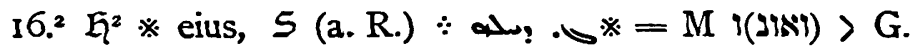
Aber S A KS (i. T.) aurov.

23. $*$ si fuerit inundatio non timebit $\mathfrak{L}^{2} *$ securus est $*$ cum illisus fuerit Jordanis in os eius, 5 (i. T.) (201 הן יעשק נהר ולא יחפוז יבטח כי־יגיח ירדן אל-פיהו. In $\mathcal{K}$ fehlt nur das in $S$ s. * Stehende. Der erste ※ ist also zu früh gesetzt. Oder soll dieser $*$ besagen, dass

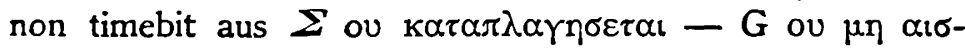




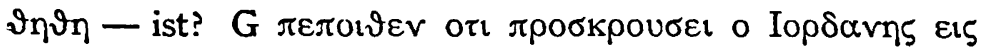

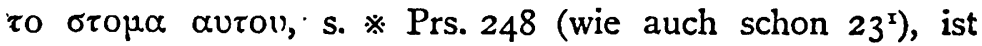
aus $\Theta$.

24. in oculo suo $\mathfrak{L}^{\mathrm{x}}$ ※ suscipiet eum torto vertice pertundit

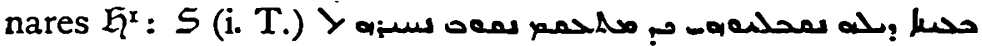

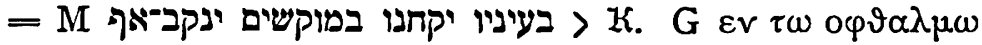

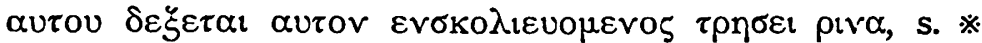
Prs. 248, ist nach $\kappa$ Reg. un. Olymp. $S$ aus $\Theta$.

26. ${ }^{x} *$ si $\mathfrak{L}^{\mathrm{x}}: \operatorname{ligabis}^{\mathrm{x}}$ circulum in ore eius, $\mathcal{S}$ (i. T.) $* .2$ התשים אגמון באפו

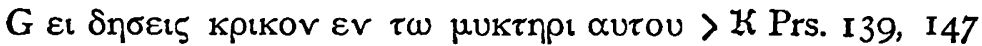
ist nach $S$ aus $\Theta$. $\mathfrak{L}$ in ore eius!

$31 .^{2} *$ et in navibus piscatorum caput eius $=M$ ובצלצל דגים

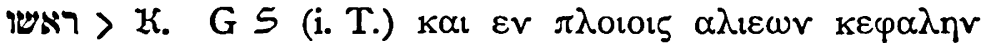

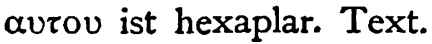

\section{Kap. XLI.}

4. $*$ non tacebo propter eum $\mathfrak{K}^{2} *$ et sermo virtutum miseretur aequalis sui $\mathfrak{L}^{x}: S$ (i. T.) hat nur den Anfang

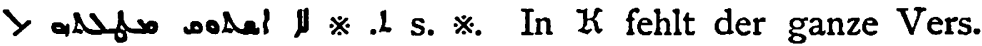

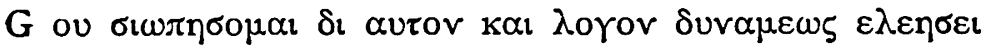

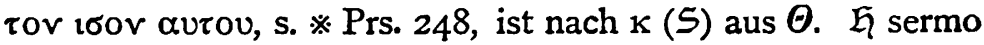

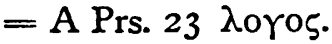

8. unus uni $*$ adhaeret et spiritus non pertransibit eum: S (i. T.) אחד באחד ינשו ורוח L לאייבא ביניהם. In $K$ fehlt nur der in $5 \mathrm{~s}$. ※ stehende $8^{x}$. G

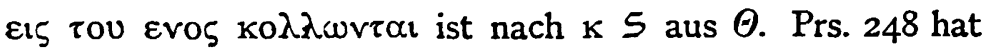
den ganzen Vers 8 s. $*$.

9. $\mathfrak{L}^{x} *$ vir fratri suo adglutinabitur: $\mathfrak{L}^{2} *$ continebuntur

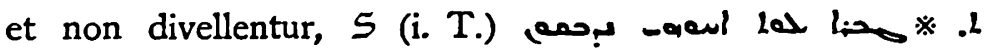

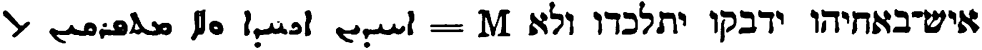

× $\mathfrak{q}^{2} *$ sigillabis. 


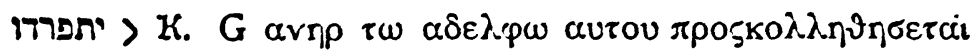

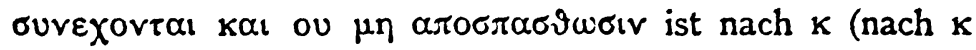
eigtl. nur $\left.9^{2}\right) S$ aus $\theta$.

$15 .^{2} \mathfrak{L}^{\mathrm{x}} *$ circumfusae ei et immobiles, $S$ (i. T.) : ע $\varepsilon \pi$ aurov ou $\sigma \alpha \lambda \varepsilon v \vartheta \eta\rceil \varepsilon \tau \alpha$, s. * Prs. 248 (wie auch schon

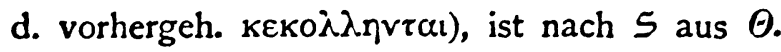

$18 .^{2} *$ hastae elatio et thoracis $\mathfrak{L}^{\mathrm{r}}:=\mathrm{M}$ חנית מסע ושריה > K. G S (i. T.) Sopv (C S $\mathrm{S}^{c .2} 23$ Prs. Ald. Compl. 5

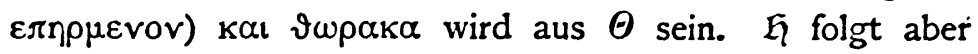

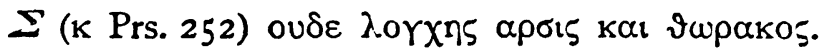

$24 .^{2} *$ computavit abyssum quasi $\left(\xi^{2}\right.$ in) deambulacrum $S$ (i. T.) > יחשב תהום

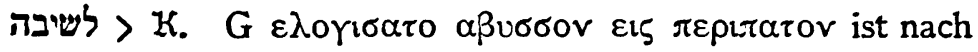
$\kappa$ aus $\Theta$. $\mathfrak{h}$ quasi $=C$ Prs. 23,252 w与. $\mathfrak{L}^{2}$ quasi in ist des Guten zu viel.

Kap. XLII.

3. $\mathfrak{Y}^{x}-$ magna et mirifica quae non noveram, $S$ (i. T.)

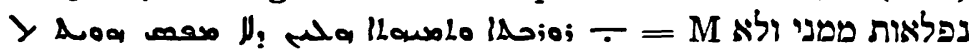
עד. Der - sollte in 5 , wie vielleicht wirklich in $\mathfrak{K}$, nur auf die Worte $\mu \varepsilon \gamma$. $k \alpha$ in $G$ sich beziehen, da נפ von G durch 2 Worte wiedergegeben ist, wovon eins zuviel.

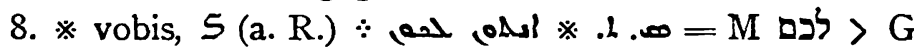
$S$ (i. T.), $K$ freilich NHTN.

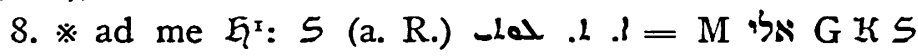
(i. T.) Prs. $249 \pi \rho \circ \zeta \varepsilon$ ist nach $S$ aus $A \Theta$.

16. $\mathfrak{L}^{\mathrm{x}}$ - et omnes anni vitae eius fuerunt CCXLVIII, $S$

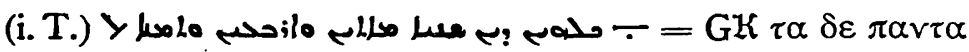
$\varepsilon \tau \eta \varepsilon \zeta \eta \sigma \varepsilon \vee \delta \imath \alpha \kappa o \sigma \alpha \tau \varepsilon \sigma \sigma \varepsilon \rho \alpha \kappa o v \tau \alpha>M$ Compl. G CCXLVIII

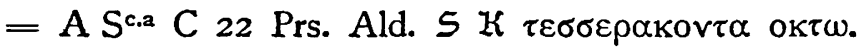

16. $\mathfrak{L}^{2} *$ et vidit Job filios suos $*$ et filios filiorum suorum

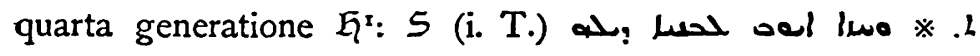




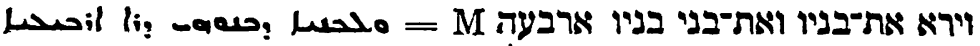

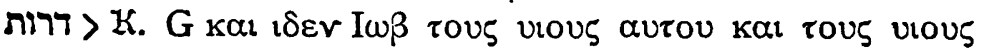

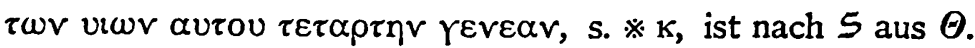

Das + G Ks nach Vers $I 7$ steht in $\mathcal{G}$ scriptum est bis

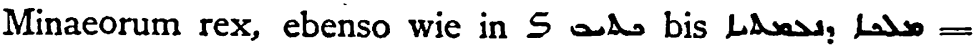

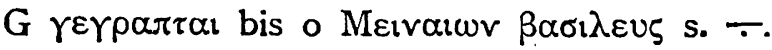

\section{Schluss.}

Fasse ich die Resultate der obigen Darlegungen kurz zusammen, so lässt sich etwa folgendes sagen:

I. Die Asterisken stehen in $\mathfrak{G}^{2}$. häufiger als in $\mathfrak{G}^{x}$, und zwar ist das Verhältnis ungefähr so, dass $\mathfrak{L}^{2}$ ca. $75 \mathrm{mal}$ allein $※, \mathfrak{G}^{\mathbf{x}}$ ca. $25 \mathrm{mal}$ allein $*$ hat, das Zahlenverhältnis der je nur in $\mathfrak{h}^{2}$ oder $\mathfrak{h}^{x}$ stehenden Asterisken also wie $3: I$ ist. Verschwindend klein ist die Zahl der $\div$, die jede der beiden Handschriften für sich hat, auch hier ist der Vorzug auf Seiten von $\mathfrak{h}^{2}$. Zuweilen sind die in Rede stehenden Asterisken Fehler für $\div$ z. B. $\mathfrak{G}^{\mathrm{x}}$ 7, II. IO, I. I2, 2 I. 34, 8; $\mathfrak{Y}^{2}$ I 4, 5; oder direkt ungehörig z. B. 9, 25. 31, 35 .

Die wertvollere von beiden Handschriften ist hinsichtlich der hexaplarischen Zeichen $\mathfrak{G}^{2}$.

2. Im Grossen und Ganzen hat $\mathfrak{L}$ weniger hexaplarische Zeichen als 5 . In einer Reihe von Fällen hat $\mathfrak{L}$ allein $\mathfrak{S}$ gegenüber $*$, sei es, dass in 5 nur der in $\mathfrak{L}$ gesetzte $*$ z. B. I $5,27.21,23.28,5.30,3.34$, I I. 40,2 , oder die ganze asteriszierte Stelle fehlt z. B. 1, 6. 1, 16. 2, 8. 2, 13. 3,3 , oder in $\mathfrak{L}$ der $*$ für einen grösseren Passus als in $\mathfrak{S}$ gilt 2. B. 21, 21. 22, 20. 23, 9. 41, 4. Falsch steht der 
* in $\mathfrak{Y}$ z. B. 3.6. $5,22.9,25.12,14.22,28.31,19.31,35$. 33, 23. 36, 27; der $*$ in $\mathfrak{Z}$ ist Fehler für - z. B. 7, 11 . 10, 1. 14, 5 I 5, I I. 34, 8. 35, 2. 39, 26. Weniger häufig sind die Stellen, in denen $\mathfrak{h}$ allein gegenüber 5 richtig - setzt, z. B. 3, I 1. 20, 7. In der weitaus grösseren Zahl der Fälle ist der - in $\mathfrak{K}$ verglichen mit $\mathfrak{S}$ an falscher Stelle gesetzt, oder überhaupt unrichtig, oder Fehler für $* z$. B. 1,18. 1, 22. 3, 10. 5,9. 14, 22, 16, 15. 19,8. 22, 29. 29, 12. 38, 5 .

$\mathfrak{h}$ ist ärmer und ungenauer hinsichtlich der haxaplarischen Zeichen als 5 . Das schliesst nicht aus, dass $\mathfrak{G}$ zuweilen bessere Angaben betreffs der hexaplarischen Zuthaten macht als 5 .

3. In den allermeisten Fällen folgt $\mathfrak{h}$ in den asteriszierten Stellen dem Texte von $\Theta$. Die aus $\Theta$ übernommenen Ergänzungen verhalten sich zu denen aus $\Sigma$ oder $A$ ungefähr wie 10 bzw. 12: I. Stellen, in denen $\mathfrak{L}$ dem Texte von $\Sigma$ folgt, sind z. B. 5, II. 9, 22. I I, 10. 14, 2 I. 2 I, IO. 30, 4. 36, 29. 37, 13. 4I, 18; einen grösseren Abschnitt hat $\mathfrak{h}$ aus $\Sigma$ aufgenommen $32,13-17$. Stellen aus $A$ sind z. B. 1, 6. 3, 5. 5, 10. 12, 10. 22,6 . 31, 17. $37,3.38,32$. Zuweilen giebt $\mathfrak{h}$ einen aus den späteren griechischen Übersetzungen gemischten Text z.. B. aus $\Theta \Sigma$ 18, 17. 19, 29. In einer Reihe von Fällen lässt sich die Quelle der hexaplarischen Einschübe für $\mathfrak{G}$ noch nicht feststellen, da in $\mathfrak{S}$ und den hexaplarische Zeichen tragenden griechischen Handschriften die betreffenden Stellen anonym überliefert sind, oder der Asteriskus überhaupt fehlt, z. B. I, I3. 2, 8. 2, I3. 3, 3. 5, 23. 7, 15. 11, 5. 12, 11. 13, 2. 13, 10. 13, 20. 16, 16. 18, 9. 19, 15. 19, I 7. $23,8.24,5.30,3.34,8.34$, I I. 34 , 18. 40, 16. Es scheint, als ob sich nähere Beziehungen zwischen den asteriszierten Stellen $\mathfrak{F}_{\mathrm{s}}$ und dem Texte von Prs. 249 finden; vgl. z. B. 1, 16. 2, 7. 2, 10. 2, 13. 3, 3. 3, I8. 5, I1. 5, 23. 9, 34. IO, 5. I2, 14. I4, 21. I4, 22. I 5, 3. I8, I3. 
I9, I 5. 22, 3. 22, 6. 23, II. 24, I. $34,22.34,26.35,3$. 39, 18. 39, 23. 40, 16. $42,8$.

$\mathfrak{h}$ giebt im Allgemeinen in seinen asteriszierten Stellen den Text $\Theta$ s wieder, weniger häufig folgt er $\boldsymbol{\Sigma}$ und $\boldsymbol{A}$.

Die auf $\mathfrak{L}$ bezüglichen Untersuchungen verfolgen in erster Linie den Zweck, einen Textzeugen für den Umfang der ursprünglichen LXX zu Hiob zu gewinnen. Für die Feld- Wald- und Wiesenkritik mag die Swete'sche Handausgabe des griechischen Hiob genügen, für ernstere Studien reicht sie nicht aus. Hat doch Swete nicht einmal die aus $\Theta$ in die gewöhnlichen griechischen Handschriften eingedrungenen Verse und Versteile in seiner Ausgabe gekennzeichnet, was doch nach dem Vorhandensein von Field's Hexapla nicht allzu schwierig gewesen wäre. Hoffentlich erfüllt die von Swete geplante grössere LXX-Ausgabe die auf sie gesetzten Hoffnungen auch nach der eben hervorgehobenen Seite hin. Vor allem wird der Herausgeber die Handschriften Prs. 248 und Colbertinus 1952 für Hiob auszubeuten haben. Im Allgemeinen wird $\mathfrak{h}$ unter den Versionen für die Gewinnung der Graeca vetus erst an dritter Stelle nach $\mathfrak{K}$ und $S$ in Betracht kommen. Meine Einzelausführungen wollen als bescheidene Vorarbeit. für die Rekonstruktion der vororigenistischen LXX zu Hiob aufgefasst sein.

In zweiter Linie gelten die Studien über $\mathfrak{G}$ der Geschichte der LXX überhaupt. In dieser Hinsicht wird noch der Frage näher zu treten sein, welchen griechischen Handschriften schliesst $\mathfrak{h}$ sich an? Damit greift aber die Untersuchung zugleich auf die erste Frage nach der Stellung Ess zur "echten" LXX zurück. Erst wenn beide Fragen erledigt worden sind, wird sich ein annähernd abschliessendes 
Urteil über den Wert von $\mathfrak{h}$ abgeben lassen. Dabei wird auch das Verhältnis von $\mathfrak{h}$ zur Vulgata mit in Betracht $z u$ ziehen sein. So sehr wir auch dem Vielschreiber Hieronymus für seine Bemühungen um den hebräischen und griechischen Bibeltext zu Danke verpflichtet sind, das Urteil, das Augustin über ihn fallte - quod Hieronymus nescit nemo scit - wird sich auch für des Hieronymus lateinische Übersetzung des griechischen Hiob als eine arge Überschätzung der Kenntnisse und Verdienste des Hieronymus herausstellen.

Das oben über $\mathfrak{q}$ Gesagte bezieht sich nur auf die beiden mit hexaplarischen Zeichen versehenen Handschriften $\mathfrak{G}^{x}$ und $\mathfrak{G}^{2}$, sobald neue, bzw. bessere Handschriften entdeckt werden, wird das abgegebene Urteil zu ergänzen oder $z u$ berichtigen sein - dass $\mathfrak{g}$ dann aber um eine Stelle als Textzeuge für die Kenntnis der Graeca vetus, etwa vor 5 , heraufrücken sollte, bezweifle ich. Wird es je dazu kommen, dass wir eine kritische Ausgabe von des Hieronymus Übertragung des griechischen Hiob erhalten? 